\title{
A Multi-Scale Model of Dendritic Cell Education and Trafficking in the Lung: Implications for T Cell Polarization
}

\author{
DAVID J. KLINKE II ${ }^{1,2}$ \\ ${ }^{1}$ Department of Chemical Engineering, West Virginia University, P.O. Box 6102, Morgantown, WV 26506-6102, USA; and \\ ${ }^{2}$ Department of Immunology, Microbiology, \& Cell Biology, West Virginia University, Morgantown, WV 26506, USA
}

(Received 13 September 2006; accepted 11 April 2007; published online 25 April 2007)

\begin{abstract}
Health Sciences face a significant challenge in translating basic science data into improved understanding of innate and adaptive immunity. To improve understanding of the dynamic role of dendritic cells in the lung, a mathematical model was developed using a physiologically structured framework that explicitly accounts for functional heterogeneity. As sentinels of the immune system, dendritic cells play critical roles in coupling innate to adaptive immunity and produce an important immune regulatory cytokine: IL-12. The term IL-12 actually refers to the net bioactivity of three related proteins; IL12p40, IL12p70, and IL12(p40) ${ }_{2}$; assembled from two independent gene products: p35 and p40. The model for dendritic cell education and trafficking was created by incorporating five dimensions: chronological time, maturational age, p35 signal, p40 signal, and spatial location. The computational framework was calibrated to and validated against appropriate experimental studies. Using this validated model, I explore the impact of dynamic changes in the lung epithelium of IL-4, IFN- $\gamma$, and PGE2 on the dynamic ability of dendritic cells to polarize $\mathrm{T}$ cell subsets. In summary, this multi-scale model provides an essential aid in understanding the impact of a dynamically changing lung microenvironment on the ability of dendritic cells to orchestrate adaptive immunity.
\end{abstract}

Keywords-Ordinary differential equations, Antigen presentation, Immunosurveillance, Cellular heterogeneity, Translational research.

\section{ABBREVIATIONS \\ DC dendritic cells \\ BM blood monocytes \\ BD blood dendritic cells \\ LD lung dendritic cells}

Address correspondence to David J. Klinke II, Department of Chemical Engineering, West Virginia University, P.O. Box 6102, Morgantown, WV 26506-6102, USA. Electronic mail: david.klinke@ mail.wvu.edu

\section{INTRODUCTION}

The technological advances in molecular biology during the 20th century provided immense insight into the individual components of complex biological systems. ${ }^{1}$ Yet, we still understand relatively little about the in vivo behavior of these integrated systems. In parallel, research costs associated with these new techniques have escalated dramatically but the commercialization rate of new therapeutic options has failed to keep pace. ${ }^{10}$ Thus, one of the main challenges facing health sciences is translating a deluge of basic science data into novel and efficacious therapeutics.

Historically, engineering is a field in which basic research is translated into commercially viable products and processes. A fundamental pillar in this field is the use of computational frameworks for interpreting and predicting the behavior of complex systems. ${ }^{41} \mathrm{By}$ applying their quantitative skillset, engineers can provide immense value by translating data into quantitative knowledge about biological systems. In particular, this approach can aid in understanding the implications of dynamic phenomena ${ }^{29}$ and identify knowledge gaps in the collective scientific understanding. ${ }^{30}$ To illustrate this approach, basic science data will be integrated into a computational framework to aid in understanding the dynamics of primary immune sensitization in the human lung.

Within the context of human immunology, defense against invading pathogens is an emergent behavior of a collection of heterogeneous cell subsets and typifies a complex system. ${ }^{14}$ Individually, each of these subsets have unique roles in orchestrating an immune response. Yet, characterizing these cell subsets in isolation provides limited insight into system behavior. Together, these cell subsets integrate information across a range of spatial and temporal timescales. Through coordinated interaction between these diverse cell subsets, evolution has shaped the immune system to respond innately to common features of pathogens 
(innate immunity) ${ }^{36}$ and to respond adaptively to novel features of pathogens (adaptive immunity). ${ }^{50}$ This paper will focus on a cell subset that connects innate and adaptive immunity - dendritic cells (DC) - in one of the most important organs for immunosurveillance - the lung.

In the following sections, relevant background information will be presented and a computational framework will be developed that represents important aspects of the education of DC in the human lung epithelium and their dynamic impact on $\mathrm{T}$ cell differentiation via Interleukin-12 (IL-12). In particular, this framework will integrate the dynamic heterogeneity of DC populations with the effect of biochemical stimuli on DC-derived IL-12 bioactivity. I focus on IL-4, IFN$\gamma$, and PGE2 as prototypical stimuli within the microenvironment of the lung epithelium. The resulting model will be calibrated to and validated against existing experimental data. Finally, I will illustrate using this model to explore how dynamic changes in the biochemical profile of the epithelial microenvironment influences $\mathrm{T}$ cell polarization within the corresponding draining lymph node.

\section{BIOLOGICAL BACKGROUND}

As the sentinels of the immune system, DC play an important role in initiating and maintaining adaptive immune responses through antigen capture and presentation, costimulation, and mediator release..$^{2,55} \mathrm{DC}$ are a dynamic population wherein subsets within the whole population exhibit different functional properties. $^{5,7,58}$ Unlike other professional antigen presenting cells (e.g., macrophages and B cells), DC exhibit a unique ability to perform different functions in the peripheral tissue and lymphoid organs. In the peripheral tissues, immature DC are "educated" through the integration of extracellular instructional stimuli (i.e., "danger signals") provided by invading pathogens and inflammatory mediators. ${ }^{35}$ Some examples of inflammatory mediators include Interleukin-4 (IL-4), Interferon- $\gamma$ (IFN- $\gamma$ ), and Prostaglandin E2 (PGE2). Through this educational process, DC become programmed to secrete a particular profile of mediators dependent on the specific immune response needed to rebuff a pathogenic insult. Maturation of DC result in the migration of these "educated" DC into the lymphoid organs where they initiate an appropriate immune response. ${ }^{19,24}$ A generalized schematic of this process is shown in Fig. 1.

An important mediator produced by mature DC that promotes the expansion of effector $\mathrm{T}$ cells and the activation of Natural Killer (NK) cells against intracellular pathogens is Interleukin-12 (IL-12). ${ }^{23}$ IL-12 is an important immune regulatory cytokine and a member of a small family of heterodimeric cytokines. ${ }^{51}$ The term IL-12 is typically used to refer to a collection of IL-12-related proteins. The bioactive form of IL-12 is a $75 \mathrm{kDa}$ heterodimer (IL12p70) formed by the disulfide-linkage of two independently regulated gene products: a $40 \mathrm{kDa}(\mathrm{p} 40)$ subunit and a $35 \mathrm{kDa}(\mathrm{p} 35)$ subunit. ${ }^{37}$ The IL12p70 heterodimer binds to naive CD4 + T cells via a heterodimeric receptor (IL-12R). ${ }^{43}$ The p40 subunit exists extracellularly as a monomer (IL12p40) or dimer (IL12(p40) 2 ). Both the monomer and dimer can block (antagonize) the action of the bioactive IL12p70. ${ }^{32}$ The appropriate cellular response is determined largely by the total number of IL-12 receptors bound by IL12p70 (IL-12 bioactivity). Sufficient signaling through the IL-12 receptor leads to polarization of naive CD4 $+\mathrm{T}$ cells towards a Th1 phenotype. ${ }^{21}$ To represent this phenomenon quantitatively, a mathematical model for IL-12 bioactivity has been created by incorporating the production of IL12p70, IL12p40, and IL12(p40) 2 by mature human DC and the interaction of these species with the IL-12 receptor. $^{30}$

\section{METHODS}

\section{Model Formulation}

The biology related to the education and trafficking of dendritic cells in the lung epithelium is shown schematically in Fig. 1. The relevant biological phenomena span molecular-level interactions through population-level behaviors. A multi-scale model is a term used to describe computational approaches that span ranges of length and spatial scales. ${ }^{56}$ To integrate related biological data from the scientific literature, I developed a multi-scale computational framework for DC education and trafficking comprised of five dimensions: chronological time, maturational age, p35 signal, p40 signal, and spatial location. In representing these five dimensions, the following sections will focus on three aspects of this biological system: DC trafficking, DC education in the lung epithelium, and DC polarization in the draining lymph nodes.

\section{Modeling DC Trafficking}

This model of the trafficking of DC focuses on representing the dynamics of the myeloid-derived DC population, as myeloid DC play a major role in the human lung in coordinating immunity under chronic and acute inflammatory conditions. ${ }^{22}$ A model for DC population maintenance and enhancement in response to inflammatory stimuli in the lung epithelium is comprised of the regulated transport of three cell 


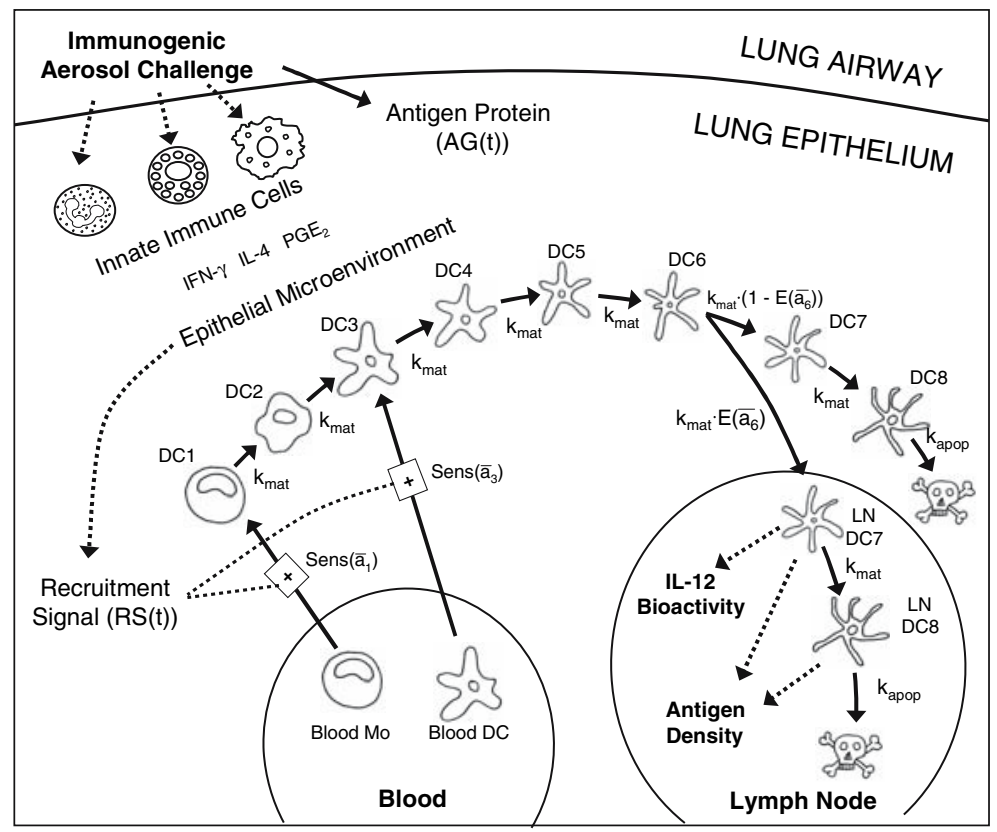

FIGURE 1. Schematic diagram of mathematical model for dendritic cell trafficking and education by the lung epithelial microenvironment. An aerosol challenge results in the increase of antigenic proteins and subsequent release of inflammatory mediators (e.g., IL-4, IFN- $\gamma$, and PGE2) by cells of the innate immune system in the epithelial microenvironment. An increase in inflammatory mediators promotes the recruitment of DC precursors from the blood into the lung epithelium. DC dynamically traffic through the lung epithelium and become programmed by the prevailing epithelial microenvironment. Upon maturation, DC migrate into the lymph nodes and present antigenic peptides obtained in the lung epithelium. In addition, mature DC produce the IL-12-related species: IL-12p70, IL-12p40, and IL-12(p40) 2. The relative production of these species by "mature" DC (LNDC 7 ) is regulated by previous exposure to regulatory mediators in the epithelial microenvironment. These three IL-12-related species competitively interact with an IL-12 receptor resulting in a net IL-12 bioactivity.

populations: blood dendritic cells (BD), blood monocytes (BM), and lung DC; and between three spatial compartments: the blood, lung epithelium, and lymphatic system. Recently, Klinke developed a model for DC trafficking and focused on capturing the dynamics in the blood and lung epithelial compartments. ${ }^{29}$ In this study, the focus of the model will be extended to incorporate the dynamics of DC trafficking into the lymph node.

DC in the lung epithelium arise from the recruitment of two precursor populations in the blood: BM and $\mathrm{BD}$. The rate of change in these blood populations can be generally represented by the following ordinary differential equation:

$$
\begin{aligned}
\frac{d \text { Blood Cell }}{d t}= & \text { Synthesis }- \text { Recruitment into lung } \\
& - \text { other losses. }
\end{aligned}
$$

The "other losses" term represents the loss in the blood cell population due to recruitment to other tissues or cell death. Using a pseudo-steady-state approximation, the "other losses" term can be expressed in terms of the steady-state synthesis and lung recruitment rates. The rate of change of the $\mathrm{BM}$ is represented by a population balance equation:

$$
\begin{aligned}
\frac{d B M(t)}{d t}= & B M_{\mathrm{syn}}-k_{\mathrm{BM}} \cdot R S(t) \cdot B M(t) \\
& -\left[B M_{\mathrm{syn}} / B M_{\mathrm{tot}}-k_{\mathrm{BM}} \cdot R S\left(t_{\mathrm{SS}}\right)\right] \cdot B M(t),
\end{aligned}
$$

where $B M_{\text {syn }}$ is the synthesis rate of new $\mathrm{BM}, B M_{\text {tot }}$ is the steady-state total blood monocyte population, $R S(t)$ is the instantaneous recruitment signal, $k_{\mathrm{BM}}$ is the sensitivity of BM to the levels of the recruitment signal, and $R S\left(t_{\mathrm{SS}}\right)$ is the steady-state lung recruitment signal. As described in more detail by Klinke, ${ }^{29}$ the recruitment signal, $R S(t)$, represents the combined dynamic effect of the introduction and elimination of an environmental insult, release, and elimination of pre-synthesized mediators (e.g., histamine from mast cells), and mobilization and endocytosis of adhesion molecules on the endothelial surface (e.g., P-selectin). While the recruitment process is generally well characterized, it is difficult to estimate the magnitude of this recruitment signal. For simplicity, the steady-state value of the recruitment signal, $R S\left(t_{\mathrm{SS}}\right)$ was assigned a value of 1 . The rate of expression and duration of 
$R S(t)$ is representative of rapidly expressed adhesion molecules that promote DC recruitment like P-selectin. Similarly the rate of change of the BD population is given by

$$
\begin{aligned}
\frac{d B D(t)}{d t}= & B D_{\mathrm{syn}}-k_{\mathrm{BD}} \cdot R S(t) \cdot B D(t) \\
& -\left[B D_{\mathrm{syn}} / B D_{\mathrm{tot}}-k_{\mathrm{BD}} \cdot R S\left(t_{\mathrm{SS}}\right)\right] \cdot B D(t) .
\end{aligned}
$$

Upon entering the lung epithelium, monocytes are assumed to become a subset of lung DC (LD). As BM, $\mathrm{BD}$, and $\mathrm{LD}$ are all defined based on different compartments, the scaling factors $L D_{\text {tot }}, B M_{\text {tot }}$, and $B D_{\text {tot }}$ are introduced to account for the different compartment sizes. These scaling factors are defined as the total number of $\mathrm{LD}, \mathrm{BM}$, and $\mathrm{BD}$, respectively.

While $\mathrm{BM}$ and $\mathrm{BD}$ are represented as an averaged population, DC within the lung epithelium require a more complex representation to account for functional heterogeneity within this compartment. The DC population in the lung epithelium is represented by a physiologically structured model. ${ }^{29}$ Physiologically structured models integrate mechanistic information at the individual level to give predictions of the behavior at the population level. The characteristics of each individual in a population are described by a set of physiological variables representing the internal and external characteristics of the individual. In this study, maturational age, antigen loading, and educational state are the defining physiological variables of DC. The state of an individual can be defined by assigning particular values to each of these the physiological variables.

Using this physiologically structured mathematical framework, a specific model for DC trafficking in the lung epithelium was created with eight equal maturational-age based subpopulations. A schematic diagram of this model is shown in Fig. 1, where the $i$ th DC subpopulation is labeled by $D C_{i}$. A generalized relationship for the rate of change in $D C_{i}$ is described by

$$
\begin{aligned}
\frac{d D C_{i}}{d t}=k_{\mathrm{mat}} \cdot\left[D C_{i-1}(t)-D C_{i}(t)\right]+R S(t) \\
\cdot \operatorname{Sens}\left(\overline{a_{i}}\right) \cdot B P_{\bar{a}_{i}}(t)-k_{\mathrm{mat}} \cdot E\left(\overline{a_{i}}\right) \cdot D C_{i}(t),
\end{aligned}
$$

where $k_{\text {mat }}$ is the DC maturation rate and the same for all $D C_{i}, R S(t)$ is the recruitment stimuli, $B P_{\overline{a_{i}}}(t)$ is the blood precursor population that gets recruited into $D C_{i}, \operatorname{Sens}\left(\overline{a_{i}}\right)$ is the sensitivity of $B P_{\overline{a_{i}}}(t)$ to the recruitment stimuli, and $E\left(\overline{a_{i}}\right)$ is the average emigration probability for $D C_{i}$ to the draining lymph node. A summary of the model variables is found in Table 1. Parameter values related to DC trafficking for each of the eight equal subpopulations are found in Tables 2 and 3 . The average emigration probability is derived from the degree of down-regulation of tissue homing and up-regulation of lymphatic homing chemokine receptors as a function of maturational age. ${ }^{48}$ Values for $E\left(\overline{a_{i}}\right)$ are shown in Table 3.

Parameter values were chosen based on appropriate measurements in humans if available. However, in the absence of specific kinetic measurements in humans, data obtained from rat or mouse studies were used. Using the set of ordinary differential equations, the estimates for unknown parameter values were determined using appropriate experimental data and guided by a parameter identification analysis. The model equations were encoded and evaluated in MatLab V7.0 (The MathWorks, Natick, MA). Summed squared error between experimental and simulated measurements was used to determine goodness-of-fit.

\section{Modeling DC Education by the Epithelial Microenvironment}

Using this age-structured model of the DC population, the lung DC can be partitioned based on the functional ability of the different subpopulations. Partitioning of the education process onto the DC subsets illustrates the unique capability of an agestructured model in connecting individual- to population-based behavior. The work in modeling IL-12 bioactivity by Klinke ${ }^{30}$ implicitly lumped the temporal and spatial separation between signal integration in the lung epithelium and biological output in the lymph node. In this study, the IL-12 bioactivity model by Klinke $^{30}$ is expanded by explicitly distributing signal integration and biological output across DC subsets. Dynamic exposure to cytokines within the epithelial microenvironment educates individual DC subsets to produce the constituent proteins of bioactive IL-12. ${ }^{25}$ The level of education, or educational state characteristic, will be represented by two additional independent variables.

In the DC trafficking model by Klinke, ${ }^{29}$ the population of DC in the lung was described by two independent variables: maturational age and chronological time. The population of DC in the lung epithelium at a given point in time is then defined in terms of the density of DC with maturational ages between $a$ and $a+d a(D C(t, a) d a)$. In this study, two additional independent variables are incorporated into the physiologically structured framework to represent the education of the DC in the lung epithelium. These two new independent variables are " $p 35$ signal" and " $p 40$ signal." These new variables represent extent of epigenetic events that result in the cumulative education of a DC at a particular point in time and maturational 
TABLE 1. List of model variables.

\begin{tabular}{|c|c|c|}
\hline Variable name & Definition & Units \\
\hline$A G(t)$ & Antigen peptide concentration in the lung epithelium & peptide $\mathrm{cm}^{-3}$ \\
\hline$A G_{D C i}(t)$ & Total internalized antigen peptides in DC subset $i$ & peptide \\
\hline$B D(t)$ & Blood dendritic cells & cell \\
\hline$B M(t)$ & Blood monocytes & cell \\
\hline$D C_{i}(t)$ & Lung epithelial DC subpopulation $i$ & cell cell ${ }^{-1}$ \\
\hline$D C_{i}^{n n}(t)$ & low p35 signal and low p40 signalD $C_{i}$ subset & cell cell ${ }^{-1}$ \\
\hline$D C_{i}^{p n}(t)$ & high p35 signal and low p40 signal $D C_{i}$ subset & cell cell ${ }^{-1}$ \\
\hline$D C_{i}^{n p}(t)$ & low p35 signal and high p40 signal $D C_{i}$ subset & cell cell ${ }^{-1}$ \\
\hline$D C_{i}^{p p}(t)$ & high p35 signal and high p40 signalDC $C_{i}$ subset & cell cell ${ }^{-1}$ \\
\hline $\operatorname{IFNg}(t)$ & Lung epithelial concentration of Interferon- $\gamma$ & $\mathrm{U} \mathrm{mL}^{-1}$ \\
\hline IL4 $(t)$ & Lung epithelial concentration of Interleukin-4 & $\mathrm{U} \mathrm{mL}^{-1}$ \\
\hline IL12p40 $(t)$ & Lymph node concentration of Interleukin-12p40 & $\mathrm{pM}$ \\
\hline IL12 $(p 40)_{2}(t)$ & Lymph node concentration of Interleukin-12p40 & $\mathrm{pM}$ \\
\hline IL12p70 $(t)$ & Lymph node concentration of Interleukin-12p70 & $\mathrm{pM}$ \\
\hline IL12- & Net IL-12 bioactivity in lymph node & unitless \\
\hline \multicolumn{3}{|l|}{ Bioactivity } \\
\hline$L D(t)$ & Total lung epithelial DC & cells \\
\hline$L N D C_{i}(t)$ & Lymph node DC subpopulationi & cell $\mathrm{cm}^{-3}$ \\
\hline$L N D C_{i}^{n n}(t)$ & low p35 signal and low p40 signal $L N D C_{i}$ subset & cell $\mathrm{cm}^{-3}$ \\
\hline$L N D C_{i}^{p n}(t)$ & high p35 signal and low p40 signal $L N D C_{i}$ subset & cell $\mathrm{cm}^{-3}$ \\
\hline$L N D C_{i}^{n p}(t)$ & low p35 signal and high p40 signalLNDC ${ }_{i}$ subset & cell $\mathrm{cm}^{-3}$ \\
\hline$L N D C_{i}^{p p}(t)$ & high p35 signal and high p40 signalLNDC ${ }_{i}$ subset & cell $\mathrm{cm}^{-3}$ \\
\hline PGE2 $(t)$ & Lung epithelial prostaglandin E2 concentration & $\mathrm{nM}$ \\
\hline$R S(t)$ & Recruitment signal & unitless \\
\hline
\end{tabular}

Model variables highlighted in bold are new additions to model presented by Klinke. ${ }^{29}$.

TABLE 2. List of model parameters and values for DC trafficking.

\begin{tabular}{|c|c|c|c|c|}
\hline Parameter name & Definition & Value & Units & References \\
\hline$B D_{\text {syn }}$ & Blood dendritic cell synthesis rate & $9.13 \times 10^{5}$ & cell $\mathrm{h}^{-1}$ & 29 \\
\hline$B D_{\text {tot }}$ & Total blood dendritic cells & $9.74 \times 10^{7}$ & cell & 53 \\
\hline$B M_{\text {syn }}$ & Blood monocyte synthesis rate & $2.49 \times 10^{7}$ & cell $\mathrm{h}^{-1}$ & 57 \\
\hline$B M_{\text {tot }}$ & Total blood monocytes & $2.80 \times 10^{9}$ & cell & 44 \\
\hline$E\left(\overline{a_{i}}\right)$ & Lung DC emigration probability & see Table 3 & unitless & \\
\hline$G_{\mathrm{Ag}}$ & Antigen error function gain & 10 & unitless & 29 \\
\hline$G_{\mathrm{RS}}$ & RS error function gain & 10 & unitless & 29 \\
\hline $\boldsymbol{k}_{\text {Agdeg }}$ & Antigen decay rate constant & 0.025 & $\mathrm{~h}^{-1}$ & 54 \\
\hline$k_{\text {apop }}$ & Lung DC apoptosis rate & 0.163 & $\mathrm{~h}^{-1}$ & 29 \\
\hline$k_{\text {basal }}$ & Basal rate of RS expression & 2.77 & $\mathrm{~h}^{-1}$ & 33 \\
\hline$k_{\mathrm{BM}}$ & Blood monocyte recruitment rate & $0.88 \times 10^{-3}$ & $\mathrm{~h}^{-1}$ & 29 \\
\hline$k_{\mathrm{BD}}$ & Blood DC recruitment rate & $7.54 \times 10^{-3}$ & $\mathrm{~h}^{-1}$ & 29 \\
\hline$k_{\text {decay }}$ & Recruitment signal decay rate & 2.77 & $\mathrm{~h}^{-1}$ & 33 \\
\hline$k_{\text {mat }}$ & Lung DC maturation rate & 0.209 & $\mathrm{~h}^{-1}$ & 29 \\
\hline$k_{\text {stim }}$ & Stimulated rate of RS expression & 450 & $\mathrm{~h}^{-1}$ & 33 \\
\hline$k_{\text {uptake }}\left(\overline{a_{i}}\right)$ & Antigen uptake rate constant for $D C_{i}$ & see Table 3 & $\mathrm{~cm}^{3} \mathrm{~h}^{-1}$ & 5 \\
\hline$L D_{\text {tot }}$ & Total lung epithelial DC & $8.66 \times 10^{7}$ & cell & 29 \\
\hline LNC & Lung DC to lymph node DC conversion & $3.91 \times 10^{6}$ & cell $\mathrm{cm}^{-3}$ & \\
\hline$R S\left(t_{S S}\right)$ & Steady-state recruitment signal & 1 & unitless & 29 \\
\hline $\operatorname{Sens}\left(\overline{a_{i}}\right)$ & Sensitivity of BM and BD to $R S(t)$ & see Table 3 & $\mathrm{~h}^{-1}$ & 29 \\
\hline$t_{\text {Agon }}$ & Start of antigen challenge & 0.2 & $\mathrm{~h}$ & 29 \\
\hline$t_{\text {Agoff }}$ & End of antigen challenge & 0.5 & $\mathrm{~h}$ & 29 \\
\hline$t_{\mathrm{RSon}}$ & Start of recruitment signal & 0.1 & $\mathrm{~h}$ & 29 \\
\hline$t_{\text {RSoff }}$ & End of recruitment signal & 0.25 & $\mathrm{~h}$ & 29 \\
\hline
\end{tabular}

Parameters highlighted in bold are new or modified relative to model presented by Klinke. ${ }^{29}$.

age. These epigenetic events, such as histone modifications or methylation of promoter regions on DNA, either facilitate or silence the transcription of genes responsible for the expression of p35 or p40 proteins. Moreover, these epigenetic events independently regulate the genes responsible for the transcription of the 
TABLE 3. List of DC subpopulation specific parameter values.

\begin{tabular}{lccccc}
\hline $\begin{array}{l}\text { Dendritic cell } \\
\text { subpopulation }\end{array}$ & $\begin{array}{c}\text { Average } \\
\text { maturational age }\left(\overline{a_{i}}\right)\end{array}$ & $\begin{array}{c}\text { Sens }\left(\overline{a_{i}}\right) \\
\left(\mathrm{h}^{-1}\right)\end{array}$ & $k_{\text {uptake }}\left(\overline{a_{i}}\right)\left(\mathrm{cm}^{3} \mathrm{~h}^{-1}\right)$ & $E\left(\overline{a_{i}}\right)($ unitless $)$ & $k_{\text {edu }}\left(\overline{a_{i}}\right)\left(\mathrm{cm}^{3} \mathrm{~h}^{-1}\right)$ \\
\hline$D C_{1}$ & 0.063 & $k_{\mathrm{BM}}$ & 0.02 & 0 & 1.0 \\
$D C_{2}$ & 0.188 & 0 & 0.07 & 0 & 1.0 \\
$D C_{3}$ & 0.313 & $k_{\mathrm{BD}}$ & 0.31 & 0 & 1.0 \\
$D C_{4}$ & 0.438 & 0 & 1.00 & 0 & 0.7 \\
$D C_{5}$ & 0.563 & 0 & 0.72 & 0 & 0.95 \\
$D C_{6}$ & 0.688 & 0 & 0.43 & 0 & 0.1 \\
$D C_{7}$ & 0.813 & 0 & 0.30 & 0 & 0.0 \\
$D C_{8}$ & 0.938 & 0 & 0.17 & 0 & 0.0 \\
$L N D C_{7}$ & 0.813 & 0 & 0.0 & 0 & 0.0 \\
$L N D C_{8}$ & 0.938 & 0.0 & & 0.0 \\
\hline
\end{tabular}

p35 and p40 genes following CD40 ligation, respectively. ${ }^{37}$ As elucidation of the specific regulatory pathways is still an active area of research, we represent the impact of stimuli within the epithelial microenvironment phenomenologically, rather than mechanistically. Accumulation of the p35 signal and $p 40$ signal provide a basis for the levels of IL12p70 and Total p40 (IL12p40 + IL12(p40) 2 ) protein expression, as highlighted in Fig. 1, when the DC are exposed to CD40 ligand in the draining lymph nodes. For the purpose of illustration, I focus on the actions of IL-4, IFN- $\gamma$, and PGE2 on DC via their modulation of the p35 signal and p40 signal. It is commonly known that IL-4 is considered a Th2-promoting cytokine and IFN$\gamma$ promotes a Th1 CD $4+\mathrm{T}$ cell response. ${ }^{45}$ PGE2, a potent modulator of immune responses, has been shown protect against constriction of the airways in patients with bronchial asthma. ${ }^{42}$

A recent concept proposed regarding the response of $\mathrm{T}$ cells is leveraged to represent the education of DC: the quantal theory of immunity. ${ }^{46}$ In essence, this theory is based upon the observed heterogeneity in response to stimuli for cell populations. Individually, a cell responds to a stimulus when a sufficient number of corresponding receptors are activated. Cells that express a high number of receptors respond at lower concentrations compared to cells that express a low number of receptors. While environmental stimuli induce an all-or-nothing (i.e., binary) response at the cellular level, a graded response is observed at the population due to the heterogeneity in receptor expression. In representing the education of DC, this binary characteristic is incorporated into the physiologically structured modeling framework. While not yet observed experimentally, this binary representation may be too restrictive as it may be shown that individual DC exhibit a graded response. In such a case, DC may be represented as a continuous population or discretized into a number $(N)$ of educational substates. Populating these educational substates becomes com- putationally more difficult as the number of substates associated with a $D C_{i}$ corresponds to $N^{2}$. For the illustrative purposes of this paper, the number of DC education states will be limited to two. Experimentally, the existence and distribution of the DC subsets may be obtained via polychromatic flow cytometry ${ }^{20}$ or immunohistochemistry. ${ }^{52}$

So far, the total DC population in the lung epithelium is discretized by maturational age into eight subpopulations. These eight subpopulations $\left(D C_{i}\right)$ are highlighted in Fig. 1. To represent the two variables for DC education, each $D C_{i}$ subpopulation is discretized further into four groups:

$$
D C_{i}(t)=D C_{i}^{n n}(t)+D C_{i}^{p n}(t)+D C_{i}^{n p}(t)+D C_{i}^{p p}(t),
$$

where each group is defined by high $\left({ }^{p}\right)$ or low $\left({ }^{n}\right)$ amounts of p35 signal and p40 signal. A schematic diagram for dynamically tracking the subset groups with different propensities to produce IL-12-related species within a $D C_{i}$ subpopulation is shown in Fig. 2. The detailed equations are described in the Appendix. Parameter values related to DC education for each of the eight equal subpopulations are found in Tables 3 and 4. A contextual record of the lung epithelial microenvironment is represented by the inheritance of p35 signal and p40 signal from the groups associated with the parent subset $\left(D C_{i-1}\right)$ and to the child subset $\left(D C_{i+1}\right)$. The rate of inheritance is matched to the rate of DC maturation by $k_{\text {mat }}$. Each DC subset can also be educated based on the product of its sensitivity to educational stimuli and the prevailing epithelial microenvironment. The impact of the prevailing epithelial microenvironment, shown as the terms "Total p40 Education" and "IL12p70 Education" in Fig. 2, is represented mathematically by Eqs. (6) and (7).

Total p40 Education

$$
=f_{1}[I L 4(t), I F N-\gamma(t)]+f_{3}[P G E 2(t)]
$$




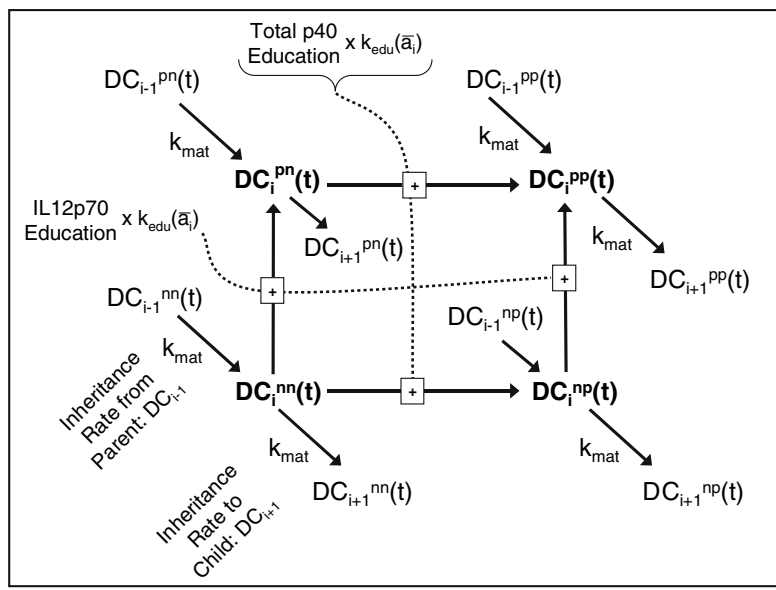

FIGURE 2. A schematic diagram of the model for dynamically tracking the accumulation of p35 signal and p40 signal within a $D C_{i}$ subpopulation. The overall education rate is the product of "Total p40 Education" or "IL12p70 Education" times the sensitivity of a DC subpopulation to response to stimuli in the epithelial microenvironment $\left(k_{\text {edu }}\left(\bar{a}_{i}\right)\right)$. A contextual record of the lung epithelial microenvironment is represented by the inheritance of p35 signal and p40 signal from the parent subset $\left(D C_{i-1}\right)$ and to the child subset $\left(D C_{i+1}\right)$.

IL12p70 Education $=f_{1}[I L 4(t), I F N-\gamma(t)] \times f_{2}[P G E 2(t)]$.

The functions $f_{1}[I L 4(t), I F N-\gamma(t)], f_{2}[P G E 2(t)]$, and $f_{3}[P G E 2(t)]$ are fully developed and described in Klinke. ${ }^{30}$ The transition between groups is modeled as irreversible based on the persistence of educational bias observed by Kalinski et al. ${ }^{26}$
In addition, the sensitivity of DC to educational stimuli in the epithelial microenvironment, similar to antigen processing ability, ${ }^{5}$ is highly dependent on maturational age. In particular, Ebner et al. showed that exposure of immature DC to IL-4 resulted in a 10fold increase in IL12p70 production compared to exposure in a mature state. ${ }^{8}$ These data provide a basis for assigning the sensitivity to educational stimuli $\left(k_{\text {edu }}\left(\overline{a_{i}}\right)\right)$ to each $D C_{i}$. I assume implicitly that $k_{\text {edu }}\left(\overline{a_{i}}\right)$ is related to the average expression levels of the receptors for IL-4, IFN- $\gamma$, and PGE2. However, to my knowledge, the relationships between cytokine receptor expression and the maturational age of myeloid DC, at a resolution similar to antigen processing ability, ${ }^{5}$ has not been reported. In turn, basal expression levels of these receptors are governed by maturational age. Values for $k_{\text {edu }}\left(\overline{a_{i}}\right)$ for each $D C_{i}$ are shown in Table 3 and are the same for all groups of a $D C_{i}$ subpopulation. As values for $k_{\text {edu }}\left(\overline{a_{i}}\right)$ were based on relative sensitivity, a scaling factor $(E S)$ was introduced to adjust the overall sensitivities to be consistent with experimental data. ${ }^{17,26,27}$ Finally, values for the dissociation constants, used in Eqs. (6) and (7), for IL$4\left(K_{D I L 4}\right)$ and IFN- $\gamma\left(K_{D I F N \gamma}\right)$ were estimated to be 4.106 and $0.368 \mathrm{U} \mathrm{mL}^{-1} \cdot{ }^{17}$ The PGE2 dissociation constant $\left(K_{D P G E 2}\right)$ was estimated from Kalinski et al. to be $0.9 \mathrm{nM}^{26}$

\section{Model of the Lymph Node Compartment}

In the process of maturation, DC alter their expression of chemokine receptors. Initially, the lung

TABLE 4. List of model parameters and values for IL-12 bioactivity.

\begin{tabular}{|c|c|c|c|c|}
\hline Parameter name & Definition & Value & Units & References \\
\hline BMRp40p70 & $\begin{array}{l}\text { Baseline molar production ratio of Total p40 } \\
(\text { IL12p40 + IL12(p40) } 2) \text { to IL12p70 }\end{array}$ & 4000 & mole mole ${ }^{-1}$ & 17,47 \\
\hline CDp70 & Baseline production of IL12p70 per high p35 signal cell & 0.204 & pg cell ${ }^{-1} \mathrm{~h}^{-1}$ & 17 \\
\hline ES & Scaling factor for DC educate rate & $1 \times 10^{-4}$ & unitless & - \\
\hline$F D$ & Fraction of Total p40 $\left(\mathrm{p} 40+(\mathrm{p} 40)_{2}\right)$ as the homodimer IL12(p40) 2 & 0.3 & mole mole ${ }^{-1}$ & 13,15 \\
\hline$G_{\text {Med }}$ & Mediator error function gain & 10 & unitless & 3 \\
\hline $\boldsymbol{k}_{\text {edu }}\left(\overline{\boldsymbol{a}_{\boldsymbol{i}}}\right)$ & $D C_{i}$ education rate constant & see Table 3 & signal $\mathrm{h}^{-1}$ & 8 \\
\hline$k_{\text {Meddec }}$ & In vivo mediator decay rate constant & 0.05 & $h^{-1}$ & 16,39 \\
\hline$K_{\text {DIL4 }}$ & Affinity constant for IL-4 & 4.11 & $\mathrm{U} \mathrm{mL}^{-1}$ & 17 \\
\hline$K_{\mathrm{Dp} 40}$ & Affinity constant for IL12p40 & 23.3 & $\mathrm{nM}$ & 32 \\
\hline$K_{\mathrm{D}(\mathrm{p} 40)_{2}}$ & Affinity constant for IL12(p40) 2 & 421 & $\mathrm{pM}$ & 32 \\
\hline$K_{\mathrm{Dp} 70}$ & Affinity constant for IL12p70 & 81.3 & $\mathrm{pM}$ & 32 \\
\hline $\boldsymbol{K}_{\text {DIFN } \gamma}$ & Affinity constant for IFN- $\gamma$ & 0.368 & $\mathrm{U} \mathrm{mL}^{-1}$ & 17 \\
\hline$K_{D P G E 2}$ & Affinity constant for PGE2 & 0.90 & $\mathrm{nM}$ & 17 \\
\hline$K_{M}$ & Half-maximal IL-12 receptor occupancy & 41.3 & sites cell ${ }^{-1}$ & 32 \\
\hline$M W_{\mathrm{p} 70}$ & IL12p70 molecular weight & 13.3 & $\mathrm{pM} \mathrm{ng}{ }^{-1} \mathrm{~mL}$ & - \\
\hline$S D$ & IL-12 receptor surface density & 2000 & sites cell ${ }^{-1}$ & 6 \\
\hline$\theta$ & DC education non-linear regression parameters & see $^{30}$ & unitless & $17,26,27$ \\
\hline$t_{\text {Medon }}$ & Start of mediator challenge & 0.2 & $\mathrm{~h}$ & 3 \\
\hline$t_{\text {Medoff }}$ & End of mediator challenge & 0.5 & $\mathrm{~h}$ & 3 \\
\hline
\end{tabular}

Parameters highlighted in bold are new or modified relative to model presented by Klinke. ${ }^{30}$. 
DC precursors, blood monocytes and blood DC, express chemokine receptors that promote their trafficking into the lung epithelium. Upon maturation, DC downregulate tissue homing chemokine receptors (e.g., CCR1, CCR5, and CCR6) and upregulate receptors that promote homing to the lymph node (e.g., CCR7). ${ }^{48}$ Consistent with this phenotypic transformation, I specify that $95 \%$ of the DC that transition from $D C_{6}$ to $D C_{7}$ exit the lung epithelium and home towards the draining lymph node. In Fig. 1, this emigration to the lymph node is represented by multiplying $k_{\text {mat }}$ by the average emigration probability for $D C_{6}\left(E\left(\overline{a_{6}}\right)\right)$. The kinetics of this process has been observed by labeling DC in the airways with fluorophore-conjugated macromolecules. ${ }^{19,54}$ These labeled DC can then be identified in the draining lymph node by flow cytometry. While multiple maturation stimuli can induce changes in chemokine receptor expression, induction of protein synthesis for the IL-12-related species is dependent on CD40 ligation, provided by $\mathrm{T}$ cells in the draining lymph nodes.

Upon interaction with $\mathrm{T}$ cells, activation of the CD40 pathway initiates the production of the IL-12related species dependent on the relative accumulation of p35 signal and p40 signal. $^{12}$ Upon activation of the CD40 pathway, the production of IL12p70 and Total p40 (IL12p40 + IL12(p40) $)_{2}$ ) have been observed to have similar kinetics. ${ }^{31}$ The production is also observed to be delayed as DC p35 and p40 mRNA expression profiles exhibit a sharp peak between 5 and $8 \mathrm{~h}$ after stimulation. In addition, protein synthesis of the IL-12-related species is transient as the p35 and p40 mRNA is completely extinguished by $18 \mathrm{~h}$. Subsequent stimulation of DC by CD40 ligand does not produce any IL-12-related species. Consequentially, DC in this state are referred to as "exhausted." 31 This phenomenon is represented by limiting the production of the IL-12-related species to the subset $D C_{7}$. Production of the IL-12-related species is implicitly modeled to depend on CD40 ligation in the lymph node. Assuming an in vitro degradation rate of $0.008 \mathrm{~h}^{-1}$, the baseline IL12p70 production (CPp70) was estimated to be $0.204 \mathrm{pg} \mathrm{h}^{-1}$ per IL12p70 positive DC $\left(D C_{7}^{p n}+D C_{7}^{p p}\right)$ based on the data of Hochrein et al. ${ }^{17}$ and kinetic measurements of Langenkamp et al. ${ }^{31} \mathrm{Gi}-$ ven the limited information regarding the relative cellular expression levels of IL12p40 and IL12(p40) $)_{2},{ }^{30}$ $30 \%$ of the Total $\mathrm{p} 40$ produced is assumed to be in the form of the dimer, IL12(p40) $)_{2}$.

To be consistent with the previous model for DC trafficking, ${ }^{29}$ each lung epithelial DC subset is normalized to the total lung epithelial DC population. The lymph node DC subsets, however, are expressed in terms of cells per $\mathrm{cm}^{3}$ of tissue. A conversion factor, $L N C$, is used to convert the lung DC population to a lymph node DC population. A lymph node that drains the conducting airways was assumed to be $1 \mathrm{~cm}^{3}$ in volume and have a population density of $1.5 \times 10^{6} \mathrm{DC} \mathrm{cm}^{-3}$. Similar densities of DC in draining lymph nodes have been reported. ${ }^{4}$ In rodents, the size of the lymph node is approximately $0.03 \mathrm{~cm}^{3}$. Changing the size of the lymph node by changing $L N C$ will not change the results presented here as they are expressed as derived intensive quantities (i.e., independent of the size of the system).

\section{RESULTS}

\section{Dendritic Cell Education}

The study by Ebner et al. ${ }^{8}$ provided guidance for assigning sensitivities of DC subpopulations to environmental stimuli. In addition, a scaling factor $(E S)$ was introduced to adjust the dynamic range of the relative sensitivities to be consistent with measurements that integrate over the entire maturational process. Three studies provided reference points for the integrated education of DC for production of the IL12-related species. ${ }^{17,26,27}$. A value for $E S$ was determined to be $1 \times 10^{-4}$ based on the observed effects of IL-4, IFN- $\gamma$, and PGE2 on IL12p70 and Total p40 $\left(\mathrm{IL} 12 \mathrm{p} 40+\mathrm{IL} 12(\mathrm{p} 40)_{2}\right)$ production by CD40-ligand stimulated DC. ${ }^{17,26,27}$ The agreement of the model results compared to the experimental data is shown in Table 5. The sensitivity of the summed squared error between the model and experimental data to changes in $E S$ is shown in Fig. 3.

\section{Dendritic Cell Trafficking to Lymph Nodes}

In representing the trafficking of DC into the lymph nodes, two additional DC maturational states were included in the model. These two DC subpopulations, $L N D C_{7}$ and $L N D C_{8}$, are represented in a different spatial compartment but are analogous to $D C_{7}$ and $D C_{8}$ in the lung epithelium. I validated the inclusion of these two lymph node DC states against two kinetic studies in mice. Ingulli and coworkers reported the kinetics of appearance of labeled DC in the draining lymph nodes following intravenous injection of antigen-pulsed DC. ${ }^{19}$ In the study by Vermaelen et al.,${ }^{54} \mathrm{a}$ fluorophore-conjugated macromolecule (FITC-OVA) was applied to the intratracheal airways. FITC-OVA was subsequently captured by endogenous DC in the airway and transported to the intrathoracic lymph nodes. Precautions were taken by Vermaelen and coworkers to ensure that the transport of FITC-OVA to the draining lymph nodes was indicative of the steady-state flux. In modeling this experiment, the value of the DC maturation rate, $k_{\text {mat }}$, was fixed at 
TABLE 5. Comparison of simulated and actual production of Total p40 (IL12p40 + IL12(p40) $)_{2}$ and IL-12p70 by CD40L-stimulated DC.

References

Experimental design

IL-4

IFN- $\gamma$

PGE2

Results

IL-12p70

Total p40 (IL12p40 + IL12(p40) $)$

\begin{abstract}
$250 \mathrm{U} / \mathrm{mL}$ $400 \mathrm{U} / \mathrm{mL}$ $100 \mathrm{nM}$
\end{abstract}

\begin{tabular}{|c|c|}
\hline $\operatorname{Exp} 1^{a}$ & 0.04 \\
\hline $\operatorname{Exp} 2^{b}$ & 1.00 \\
\hline $\operatorname{Exp} 3^{c}$ & 1.00 \\
\hline Model & 1.00 \\
\hline $\operatorname{Exp} 1^{d}$ & 1.00 \\
\hline $\operatorname{Exp} 2^{e}$ & 1.00 \\
\hline $\operatorname{Exp} 3^{f}$ & 1.00 \\
\hline Model & 1.0 \\
\hline
\end{tabular}

\section{$+$}

$-$

$-$

4.67

2.00

$-$

3.63

0.91

0.82

$-$

0.60

-
+
-

4.78

8.25

6.81

3.77

5.56

$-$

4.43

$\begin{array}{ll}+ & - \\ + & - \\ - & +\end{array}$

$\begin{array}{cll}46.7 & - & 17 \\ 28.0 & - & 17 \\ - & 0.10 & 26 \\ 35.6 & 0.10 & \text { This study } \\ 1.66 & - & 17 \\ 2.50 & - & 17 \\ - & 8.54^{9} & 27 \\ 2.07 & 8.40 & \text { This study }\end{array}$

The experimental results were reported for human monocyte-derived DC in response to co-culture with PGE2 $2^{26,27}$ and combinations of IL-4 and IFN $-\gamma{ }^{17}$ The experimental values were normalized to a basal concentrations obtained in the absence of IFN- $\gamma$, IL-4, and PGE2. Experimental values were normalized to ${ }^{\mathrm{a}} 90 \mathrm{pg} / \mathrm{mL},{ }^{\mathrm{b}} 40 \mathrm{pg} / \mathrm{mL},{ }^{\mathrm{c}} 3 \mathrm{ng} / \mathrm{mL},{ }^{\mathrm{d}} 53 \mathrm{ng} / \mathrm{mL},{ }^{\mathrm{e}} 18 \mathrm{ng} / \mathrm{mL}$, and ${ }^{\mathrm{f}} 0.25 \mathrm{ng} / \mathrm{mL}$.

${ }^{9} \mathrm{DC}$ were stimulated with TNF- $\alpha$.



FIGURE 3. The sensitivity of summed squared error for production of the IL-12-related species to changes in the value of the education scaling factor (ES). The summed squared error quantified the deviation between the predicted and measured relative enhancement of Total p40 (IL12p40+ IL12 $\left.(\mathrm{p} 40)_{2}\right)$ and IL12p70 production following exposure to combinations of IL-4, IFN- $\gamma$, and PGE2, as shown in Table 5.

$0.209 \mathrm{~h}^{-1}$, as determined previously. ${ }^{29}$ The appearance of DC labeled with FITC-OVA is reproduced in Fig. 4 and provides in vivo validation of DC trafficking from the airways to the lymph node under steady-state conditions. To provide a comparison between the study of Vermaelen et $a l^{54}$ and the model, a value for the rate constant for antigen decay $\left(k_{\mathrm{Agdeg}}\right)$ in the lung epithelium must be determined. The dynamic appearance of labeled DC in the lymph node as a function of $k_{\text {Agdeg }}$ is shown in Fig. $5 \mathrm{a}$. At a high value of $k_{\text {Agdeg }}$, labeled DC within the lymph node reach a maximum at approximately $20 \mathrm{~h}$ and disappear from the lymph node by $50 \mathrm{~h}$. As the simulation illustrates, a reduction

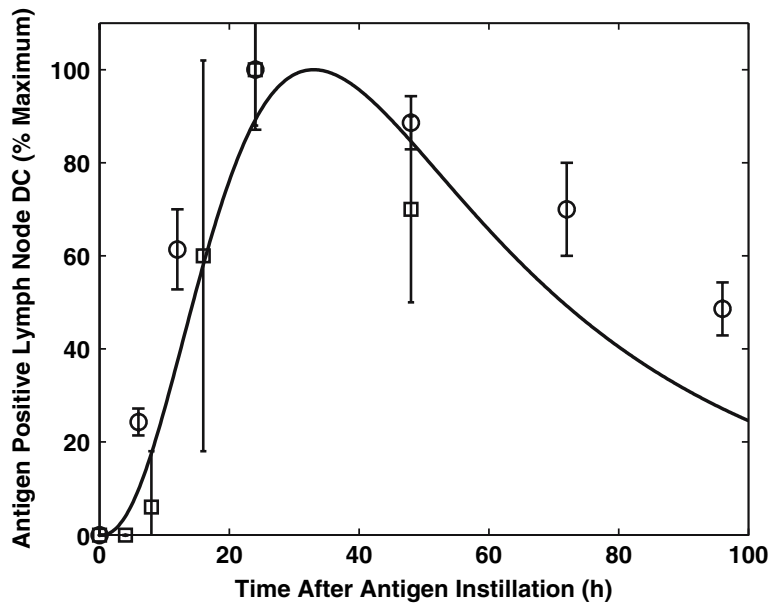

FIGURE 4. The dynamic response of antigen positive lymph node DC (Ag + LNDC) compared to the experimental results of Ingulli et al. ${ }^{19}$ (squares) and Vermaelen et al. ${ }^{54}$ (circles) for an antigen decay rate constant equal to $0.025 \mathrm{~h}^{-1}$. In the model, Ag + LNDC are comprised of subsets DC7 and DC8.

in $k_{\text {Agdeg }}$ prolongs the presence of antigen positive DC in the lymph node. Furthermore, the appearance of antigen positive DC in the lymph node is relatively insensitive to the rate of disappearance of the antigen from the lung. This observation provides another independent validation of the age-structured model developed by Klinke. ${ }^{29}$ The goodness-of-fit is quantified by a summed squared error measurement between simulated and experimental results. The fit of the model is shown as a function of $k_{\text {Agdeg }}$ in Fig. $5 b$. Using an optimal value of $0.025 \mathrm{~h}^{-1}$ for $k_{\mathrm{Agdeg}}$, the simulation appearance of labeled DC in the lymph node agrees favorably with both experimental studies. Given the single injection protocol used by Ingulli 

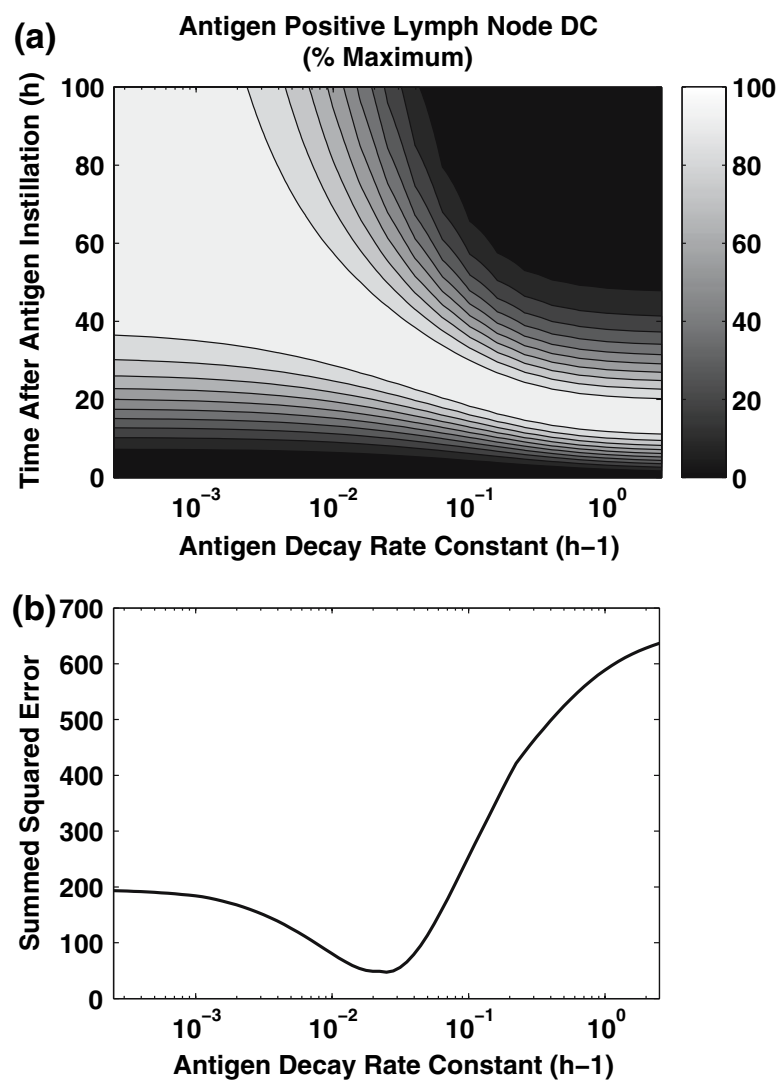

FIGURE 5. The dynamic response of antigen positive lymph node DC (Ag + LNDC) as a function of rate constant for antigen decay $\left(k_{\mathrm{Agdec}}\right)$ in the lung epithelium $(\mathrm{a})$. White on the contour plot corresponds to $100 \%$ of the maximum value observed during the simulation. The summed squared error between simulated and experimentally measured Ag + LNDC following antigen instillation as a function of $k_{\mathrm{Agdec}}(\mathrm{b})$.

et al. ${ }^{19}$ the rapid decline in labeled-DC is expected compared to the study by Vermaelen and coworkers. ${ }^{54}$ While the disappearance of FITC-labeled DC from the lymph node is assumed to be due to DC apoptosis, the FITC-label may be quenched due to acidification of FITC-OVA containing endosomes. ${ }^{11}$ In sensitized animals, exposure to FITC-OVA may also induce an inflammatory response and thereby increase $k_{\text {mat }}$. Future studies will explore this possibility.

\section{Dendritic Cell Polarization}

In the model for IL-12 bioactivity presented by Klinke, ${ }^{30}$ the production of IL-12-related species by mature DC was assumed to be constant. As demonstrated definitively by Langenkamp et al., expression of the IL-12-related species following DC maturation is a dynamic phenomenon. ${ }^{31}$ To capture this behavior, the production of the IL-12-related species was limited to one DC subpopulation, $D C_{7}$, and parameters were determined based upon this theoretical framework. In reproducing the experimental conditions of Langenkamp et al., ${ }^{31}$ it was assumed that DC were homogeneous consisting of entirely $\mathrm{DC}$ in the $D C_{5}$ subpopulation. Langenkamp and coworkers used lipopolysaccharide, a gram-negative bacterial product that activates the Toll-like receptor 4 pathway, to promote further maturation and measured the kinetics of IL12p70 production in the draining lymph node. While the addition of LPS may increase the maturation rate of DC, I assumed that $k_{\text {mat }}$ was unchanged from the value obtained from other in vivo studies. ${ }^{29} \mathrm{~A}$ comparison of the simulated and experimentally measured production of IL12p70 is shown in Fig. 6. The model simulates an earlier initiation and later extinction of IL12p70 production compared to Langenkamp et al. $^{31}$ Model agreement could be improved by increasing the number of maturational states from the current configuration of eight compartments. A residence time distribution of DC within the airways may provide guidance into an optimal number of DC subpopulations. $^{9}$

\section{DISCUSSION}

In creating a model that represents the combination of DC education and trafficking within the lung epithelium, I have integrating two sub-models that were created to capture individually the bioactivity of IL-12 and the age-structure of DC trafficking. By building upon these previous studies, this calibrated and validated multi-scale model provides a more comprehensive picture of DC biology within the lung. As mentioned above, myeloid DC are a subset of professional antigen presenting cells that also include

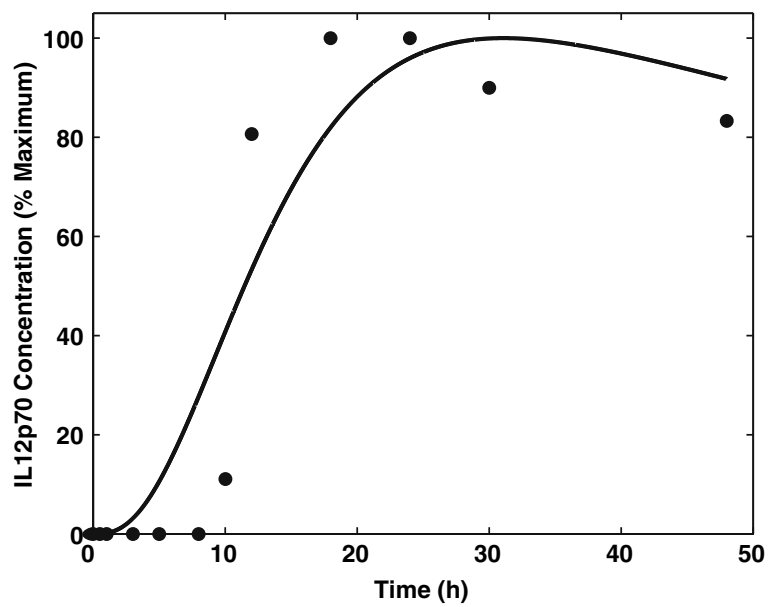

FIGURE 6. The simulated production of IL12p70 by mature DC compared to the experimental results of Langenkamp et al. $^{31}$ In the model, DC7 is the only DC subset that produces the related IL-12 species. 
macrophages and $\mathrm{B}$ cells. ${ }^{2,55}$ The functional roles of macrophages and $\mathrm{B}$ cells are largely determined by local interactions within peripheral tissues and follicular regions of the lymph nodes, respectively. In contrast, this model attempts to address global information transfer by the trafficking of myeloid DC from the peripheral tissues to the draining lymph nodes. In representing this global information transfer, DC education is intimately connected to the agestructured framework presented in the DC trafficking model. Specifically, DC education, as represented by accumulation of $p 35$ signal and $p 40$ signal, occurs in the peripheral tissue (i.e., when DC are in an immature state) and production of the IL-12-related species by previously educated DC occurs in the draining lymph nodes (i.e., when DC are in a mature state). Conversely, DC in the lung epithelium do not produce IL12-related species and cytokines in the lymph node have little impact on modulating the production of IL12-related species by mature DC. Once constructed, validated mathematical models provide unique platforms for interpreting in vivo measurements of biological systems from a dynamic, quantitative, and inexpensive perspective.

To illustrate the value of this model for interpreting in vivo studies, I will explore the implications of dynamic in vivo observations of the asthmatic disease state on DC education. The immune response to an inflammatory challenge in the lung is a complex interaction between inflammatory cells and biochemical mediators that aid in cell-to-cell communication. For the purpose of this illustration, let us consider two classes of mediators. The first class of mediators are pre-synthesized and released immediately. However, pre-synthesized mediators become quickly exhausted following an inflammatory provocation. An example of this first class is histamine, which is pre-synthesized and rapidly released from mast cell granules upon cross-linking of Fc $\varepsilon$ R 1 receptors. ${ }^{49}$ The second class of mediators require de novo synthesis when cells receive appropriate stimuli. De novo synthesis of proteins results in the delay in appearance but can be sustained for longer periods. Based on clinical observation in asthmatics, IL-4 exhibits characteristics of this second class of mediators. Besides having different dynamics, these two classes may also have different biological effects. To illustrate this concept, I assume that the first class only modulates recruitment of DC precursors into the lung epithelium. This recruitment signal is shown in Fig. 7. I also assume that the second class of mediators only modulates the education of DC in the lung. In a series of three simulations, I combine the recruitment signal with different combinations of mediators. In each of these experiments, the initial concentrations of the different mediators are equal to

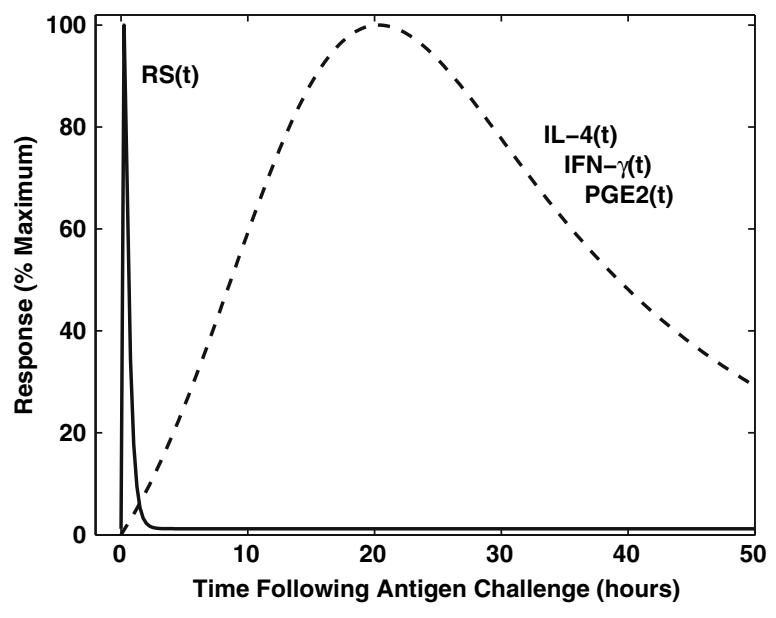

FIGURE 7. A simulated antigen challenge is comprised of a rapid increase in recruitment signal $(R S(t)$, solid curve) and a more gradual change in IL-4 concentration (dotted). IL-4 and $R S(t)$ responses are normalized to their respective maximum values. The differences in dynamics illustrate proteins that are expressed from pre-synthesized granules (e.g., $R S(t))$ or require de novo synthesis following a provocation (e.g., IL-4). This simulated response provides the input functions for the IL-12 bioactivity response shown in Fig. 8.

zero. In the first simulation, a simulated dynamic profile of IL-4 in the lung epithelium, also shown in Fig. 7, was incorporated based on the dynamic measurements by Batra et al. ${ }^{3}$ in mild atopic asthmatics. During this simulation, IL-4 reached a maximum concentration of $12.2 \mathrm{U} \mathrm{mL}^{-1}$.

The impact on DC for this combination of rapid recruitment signal and delayed expression of IL-4 is shown in Fig. 8. The slower change in IL-4 in conjunction with a rapid influx of DC precursors into the lung epithelium results in a dynamic change in the IL12-related species in the lymph node. The evolution in Total p40 and IL12p70 over a 7-day period is shown by the red curve in Fig. 8 and overlaid upon the relative IL-12 bioactivity. The value determined for IL-12 bioactivity corresponds to the relative level of proliferation in PHA-stimulated lymphoblasts treated with 2.7 pM IL12p70. The relative IL-12 bioactivity is an indication of the Th1 polarization potential elicited by mature DC in the draining lymph nodes. The steadystate IL12p70 and Total p40 concentrations in the lymph node are highlighted by the solid circle. The simulation results show that the lymph node environment becomes slightly favorable for the polarization of naive $\mathrm{CD} 4+\mathrm{T}$ cells into a Th1 phenotype by the increase in relative IL-12 bioactivity.

In the second simulation, I hypothesize that IL-4 and IFN- $\gamma$ both exhibit a similar dynamic profile, as shown in Fig. 7. In this simulation, the concentrations of IL-4 and IFN- $\gamma$ both reached a maximum of 12.2 $\mathrm{U} \mathrm{mL}^{-1}$. The impact of both IL-4 and IFN- $\gamma$ on 
Th1 Polarization Potential as Predicted by IL -12 Bioactivity

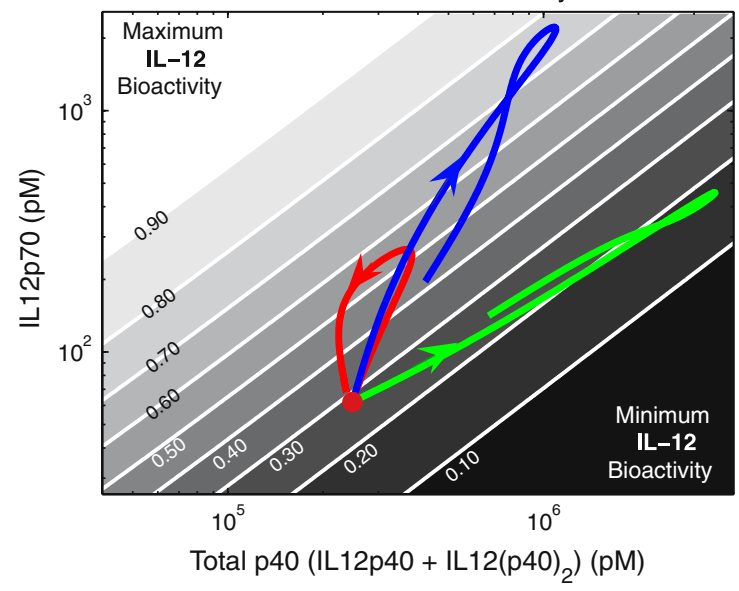

FIGURE 8. The simulated concentration of IL12p70 and Total p40 (IL12p40+IL12 $\left.(\mathrm{p} 40)_{2}\right)$ over a 7-day period in response to a simulated antigen challenge with IL-4 (red), IL-4 + IFN- $\gamma$ (blue), and IL-4+IFN- $\gamma+$ PGE2 (green). The arrows indicate the progressive change in concentrations as a function of increasing time. The steady-state levels of IL12p70 and Total p40 in the lymph node is highlighted by the solid red circle. The contour plot represents the relative IL-12 bioactivity to that induced by PHA-stimulated lymphoblasts treated with 2.7 pM IL12p70. An increase in IL-12 bioactivity is related to an increase in potential for polarization of naive CD4+ $T$ cells towards a Th1 phenotype.

the relative IL-12 bioactivity is shown by the blue curve in Fig. 8. The combination of both IL-4 and IFN- $\gamma$ significantly increases the polarization towards a Th1 phenotype. As was suggested by Hochrein et al., ${ }^{17}$ the impact of IL-4 on DC suggests an indirect promotion of Th1 polarization. In contrast, it is commonly stated that IL-4 promotes the Th2 polarization of naive CD4 $+\mathrm{T}$ cells. ${ }^{45}$ However, the Th2 polarization potential of IL-4 is primarily based upon the direct action of IL- 4 and IFN- $\gamma$ on naive CD4 + T cells. This result highlights the pleotropic nature of IL4, whereby the geographical restriction in IL-4 expression influences CD4 $+\mathrm{T}$ cell polarization.

In the third simulation, I hypothesize that IL-4, IFN- $\gamma$, and PGE2 all exhibit the same dynamic profile as IL-4. In this final simulation, the concentrations of IL-4 and IFN- $\gamma$ both reached a maximum of 12.2 $\mathrm{U} \mathrm{mL}^{-1}$, while PGE2 reached a maximum of $12.2 \mathrm{nM}$. The resulting dynamic change in the relative IL-12 bioactivity is shown by the green curve in Fig. 8 . In contrast to the presence of IL-4 or IFN- $\gamma$ alone, the addition of PGE2 inhibits the ability of IL-4 and IFN$\gamma$ to polarize naive $\mathrm{CD} 4+\mathrm{T}$ cells toward a Th1 phenotype. By reducing the bioactivity of IL-12, this simulation suggests that PGE2 may promote Th2 polarization. By focusing on the impact of PGE2 on DC education, I neglected the potential impact of PGE2 on the DC maturation rate. In vitro, PGE2 has also been shown to modulate characteristics of DC maturation including upregulation of the chemokine receptor CCR7, essential for homing to secondary lymphoid organs. ${ }^{34}$ However, the in vivo significance of this effect of PGE2 on maturation has not been demonstrated. Finally, an experimental protocol that combines an aerosol challenge of IL-4, IFN- $\gamma$, and PGE2, in various combinations, coupled with characterization of recent lung emigrants to the lymph node can validate these computational predictions. Cannulation of draining lymphatics in the lung can also be used to provide a source of these recent DC emigrants from the lung following provocation with biochemical mediators. ${ }^{38}$

\section{Opening the Immune Loop}

From an engineering perspective, the integrated immune response to pathogens can be described as an interplay between a series of subsystems. These subsystems include the innate immune cells, DC, T cells, and B cells. The first step in understanding the interplay between subsystems is to identify the structure of a particular subsystem, i.e., the causal relationships that give rise to system behavior. This paper focuses on characterizing the structure of the dendritic cell subsystem within the lung in the context of allergic asthma.

One of the most pervasive problems in the analysis of physiological systems is the identification problem. ${ }^{28}$ The identification problem is the process of specifying the causal relationships between system components consistent with a set of input and output measurements. Understanding these causal relationships is essential for the rational design of novel therapeutics. While many approaches exist for the identification of simple single-input-single-output (SISO) systems, approaches for understanding more complex closed-loop systems, like the immune system, are less well developed. Closed-loop systems are particularly challenging as it is impossible to identify the connectivity within or between subsystems based upon overall input (e.g., genetic background or environmental insult) and output (e.g., T helper cell response) measurements. Historically, the causal mechanisms underlying closedloop systems in physiology have been identified via ingenious methods for "opening the loop." A classic example of this is the discovery of insulin and it's role in connecting food intake to substrate metabolism. This landmark discovery led to the stratification of diabetics into insulin deficient (Type 1) and non-insulin deficient (Type 2) classifications.

By opening the loop, a closed-loop system is reduced to a SISO system. Opening the loop in this context refers to the dynamic measurement of internal 
states of the DC subsystem in vivo including: blood precursor populations, epithelial mediator microenvironment, and characteristics of DC that traffick to the draining lymph node. In addition, in vitro studies are also helpful in identifying more general causal relationships within the dendritic cell subsystem. By integrating these diverse data into a computational framework that explicitly states causal relationships, a new quantitative aid for interpreting in vivo studies has been created. Furthermore, a validated framework provides a basis for reverse engineering the significant pathways that underlie the emergent in vivo response. Examples of such an application include determining the contributions of memory $\mathrm{T}$ cells that reside in the lung epithelium vs. the draining lymph node. Alternatively, this validated framework could be used to interpret the impact of effector $\mathrm{T}$ cell populations on DC during primary vs. recall responses. In addition, exploration of sensitive pathways within the validated framework can provide insight into patient or species differences.

The sequence of cellular events responsible for DC trafficking is common to both CD4 + Th1 and CD4+ Th2 recall responses ${ }^{18}$ and reflects the generality of the pathogen surveillance role provided by DC in the lung. While much is known about recall responses, relatively little is known about the exact details of the initial sensitization process in vivo. Experimental studies primarily focus on the recall response to a particular antigenic protein following primary sensitization. Yet, the dynamic behavior in epithelial microenvironment may be vastly different in cases of primary vs. recall responses. During a primary response, the epithelial microenvironment reflects the innate immune response to an antigenic challenge. In contrast, a recall response includes contributions from adaptive immune components in addition to innate immune components. Ultimately, understanding this complex environment via mathematical modeling holds promise for the rational design of novel therapeutic strategies. In particular, this model could be used to optimize the timing and delivery of adjuvants to DC that reverse or dampen pre-existing immune bias. However, a more immediate goal will be to model predictively the dynamic in vivo primary response to simple combinations of stimuli as illustrated in Fig. 8.

\section{CONCLUSIONS}

The immune system is comprised of dynamic nonlinear interactions between multiple cell subsets that are heterogeneous and distributed spatially. To focus on one important cell subset that connects innate to adaptive immunity, I developed a multi-scale mathematical model for dendritic cell education and trafficking comprised of five dimensions: chronological time, maturational age, p35 signal, p40 signal, and spatial location. This model was developed by integrating diverse data into a coherent physiologically structured computational framework. This computational framework explicitly accounts for DC functional heterogeneity. In addition, the computational framework has been validated against appropriate experimental studies.

This new framework provides an aid for understanding the impact of a dynamically changing lung microenvironment on the ability of DC to polarize naive CD4 + into different effector populations by modulating the bioactivity of IL-12. Furthermore, this computational framework provides a scalable platform for further exploration of immune sensitization in the lung. Validated models, like the one presented here, are invaluable for interpreting non-intuitive system behavior and exploring possible hypotheses given the inherent difficulty in studying the human immune system. ${ }^{59}$ Ultimately, understanding the dynamics of DC education and trafficking may lead to effective therapies that modulate the immune response in a desired direction. ${ }^{40}$ The computational framework developed here is another essential step towards that goal.

\section{APPENDIX: SPECIFICATION OF MODEL EQUATIONS}

The following section describes specifically the equations used in this model. The particular model equations for $B M, B D, D C_{i}^{n n}, D C_{i}^{p n}, D C_{i}^{n p}$, and $D C_{i}^{p p}$ used in the simulations are as follows:

$$
\begin{aligned}
\frac{d B M}{d t}= & B M_{\mathrm{syn}}-k_{\mathrm{BM}} \cdot R S(t) \cdot B M(t) \\
& -\left[B M_{\mathrm{syn}} / B M_{\mathrm{tot}}-k_{\mathrm{BM}} \cdot R S\left(t_{\mathrm{SS}}\right)\right] \cdot B M(t)
\end{aligned}
$$

$$
\begin{aligned}
\frac{d B D}{d t}= & B D_{\mathrm{syn}}-k_{\mathrm{BD}} \cdot R S(t) \cdot B D(t) \\
& -\left[B D_{\mathrm{syn}} / B D_{\mathrm{tot}}-k_{\mathrm{BD}} \cdot R S\left(t_{\mathrm{SS}}\right)\right] \cdot B D(t)
\end{aligned}
$$

$$
\begin{gathered}
\frac{d D C_{1}^{n n}}{d t}=-k_{\mathrm{mat}} \cdot D C_{1}^{n n}(t)+R S(t) \cdot \operatorname{Sens}\left(\overline{a_{1}}\right) \cdot \frac{B M(t)}{L D_{\mathrm{tot}}}- \\
k_{\mathrm{edu}}\left(\overline{a_{1}}\right) \cdot E S \cdot D C_{1}^{n n}(t) \cdot(f p 40+f p 70)
\end{gathered}
$$




$$
\begin{aligned}
\frac{d D C_{2}^{n n}}{d t}= & k_{\mathrm{mat}} \cdot D C_{1}^{n n}(t)-k_{\mathrm{mat}} \cdot D C_{2}^{n n}(t) \\
& -k_{\mathrm{edu}}\left(\overline{a_{2}}\right) \cdot E S \cdot D C_{2}^{n n}(t) \cdot(f p 40+f p 70)
\end{aligned}
$$

$$
\begin{aligned}
\frac{d D C_{3}^{n n}}{d t}=k_{\mathrm{mat}} \cdot D C_{2}^{n n}(t)-k_{\mathrm{mat}} \cdot D C_{3}^{n n}(t)+R S(t) \\
\cdot \operatorname{Sens}\left(\overline{a_{3}}\right) \cdot \frac{B D(t)}{L D_{\mathrm{tot}}}-k_{\mathrm{edu}}\left(\overline{a_{3}}\right) \cdot E S \\
\cdot D C_{3}^{n n}(t) \cdot(f p 40+f p 70) \\
\frac{d D C_{4}^{n n}}{d t}=k_{\mathrm{mat}} \cdot D C_{3}^{n n}(t)-k_{\mathrm{mat}} \cdot D C_{4}^{n n}(t) \\
-k_{\mathrm{edu}}\left(\overline{a_{4}}\right) \cdot E S \cdot D C_{4}^{n n}(t) \cdot(f p 40+f p 70)
\end{aligned}
$$

$$
\begin{aligned}
\frac{d D C_{5}^{n n}}{d t}= & k_{\mathrm{mat}} \cdot D C_{4}^{n n}(t)-k_{\mathrm{mat}} \cdot D C_{5}^{n n}(t) \\
& -k_{\mathrm{edu}}\left(\overline{a_{5}}\right) \cdot E S \cdot D C_{5}^{n n}(t) \cdot(f p 40+f p 70)
\end{aligned}
$$

$$
\begin{aligned}
\frac{d D C_{6}^{n n}}{d t}= & k_{\mathrm{mat}} \cdot D C_{5}^{n n}(t)-k_{\mathrm{mat}} \cdot\left[1-E\left(\overline{a_{6}}\right)\right] \cdot D C_{6}^{n n}(t) \\
& -k_{\mathrm{mat}} \cdot E\left(\overline{a_{6}}\right) \cdot D C_{6}^{n n}(t)-k_{\mathrm{edu}}\left(\overline{a_{6}}\right) \\
& \cdot E S \cdot D C_{6}^{n n}(t) \cdot(f p 40+f p 70)
\end{aligned}
$$

$$
\frac{d D C_{7}^{n n}}{d t}=k_{\mathrm{mat}} \cdot\left[1-E\left(\overline{a_{6}}\right)\right] \cdot D C_{6}^{n n}(t)-k_{\mathrm{mat}} \cdot D C_{7}^{n n}(t)
$$

$$
\begin{aligned}
\frac{d L N D C_{7}^{n n}}{d t}= & k_{\mathrm{mat}} \cdot L N S \cdot E\left(\overline{a_{6}}\right) \cdot D C_{6}^{n n}(t) \\
& -k_{\mathrm{mat}} \cdot L N D C_{7}^{n n}(t)
\end{aligned}
$$

$$
\frac{d D C_{8}^{n n}}{d t}=k_{\mathrm{mat}} \cdot D C_{7}^{n n}(t)-k_{\mathrm{apop}} \cdot D C_{8}^{n n}(t)
$$

$$
\frac{d L N D C_{8}^{n n}}{d t}=k_{\mathrm{mat}} \cdot L N D C_{7}^{n n}(t)-k_{\mathrm{apop}} \cdot L N D C_{8}^{n n}(t)
$$

$$
\begin{aligned}
\frac{d D C_{1}^{p n}}{d t}= & -k_{\mathrm{mat}} \cdot D C_{1}^{p n}(t)+k_{\mathrm{edu}}\left(\overline{a_{1}}\right) \cdot E S \cdot D C_{1}^{n n}(t) \\
& \cdot f p 70-k_{\mathrm{edu}}\left(\overline{a_{1}}\right) \cdot E S \cdot D C_{1}^{p n}(t) \cdot f p 40
\end{aligned}
$$

$$
\begin{aligned}
\frac{d D C_{2}^{p n}}{d t}= & k_{\mathrm{mat}} \cdot D C_{1}^{p n}(t)-k_{\mathrm{mat}} \cdot D C_{2}^{p n}(t)+k_{\mathrm{edu}}\left(\overline{a_{2}}\right) \\
& \cdot E S \cdot D C_{2}^{n n}(t) \cdot f p 70-k_{\mathrm{edu}}\left(\overline{a_{2}}\right) \cdot E S \\
& \cdot D C_{2}^{p n}(t) \cdot f p 40
\end{aligned}
$$

$$
\begin{aligned}
\frac{d D C_{3}^{p n}}{d t}= & k_{\mathrm{mat}} \cdot D C_{2}^{p n}(t)-k_{\mathrm{mat}} \cdot D C_{3}^{p n}(t)+k_{\mathrm{edu}}\left(\overline{a_{3}}\right) \\
& \cdot E S \cdot D C_{3}^{n n}(t) \cdot f p 70-k_{\mathrm{edu}}\left(\overline{a_{3}}\right) \cdot E S \\
& \cdot D C_{3}^{p n}(t) \cdot f p 40
\end{aligned}
$$

$$
\begin{aligned}
\frac{d D C_{4}^{p n}}{d t}= & k_{\mathrm{mat}} \cdot D C_{3}^{p n}(t)-k_{\mathrm{mat}} \cdot D C_{4}^{p n}(t)+k_{\mathrm{edu}}\left(\overline{a_{4}}\right) \\
& \cdot E S \cdot D C_{4}^{n n}(t) \cdot f p 70-k_{\mathrm{edu}}\left(\overline{a_{4}}\right) \cdot E S \\
& \cdot D C_{4}^{p n}(t) \cdot f p 40
\end{aligned}
$$

$$
\begin{aligned}
\frac{d D C_{5}^{p n}}{d t}= & k_{\mathrm{mat}} \cdot D C_{4}^{p n}(t)-k_{\mathrm{mat}} \cdot D C_{5}^{p n}(t)+k_{\mathrm{edu}}\left(\overline{a_{5}}\right) \\
& \cdot E S \cdot D C_{5}^{n n}(t) \cdot f p 70-k_{\mathrm{edu}}\left(\overline{a_{5}}\right) \cdot E S \\
& \cdot D C_{5}^{p n}(t) \cdot f p 40
\end{aligned}
$$

$$
\begin{aligned}
\frac{d D C_{6}^{p n}}{d t}= & k_{\mathrm{mat}} \cdot D C_{5}^{p n}(t)-k_{\mathrm{mat}} \cdot\left[1-E\left(\overline{a_{6}}\right)\right] \cdot D C_{6}^{p n}(t) \\
& -k_{\mathrm{mat}} \cdot E\left(\overline{a_{6}}\right) \cdot D C_{6}^{p n}(t)+k_{\mathrm{edu}}\left(\overline{a_{6}}\right) \cdot E S \\
& \cdot D C_{6}^{n n}(t) \cdot f p 70-k_{\mathrm{edu}}\left(\overline{a_{6}}\right) \cdot E S \cdot D C_{6}^{p n}(t) \cdot f p 40
\end{aligned}
$$

$$
\frac{d D C_{7}^{p n}}{d t}=k_{\mathrm{mat}} \cdot\left[1-E\left(\overline{a_{6}}\right)\right] \cdot D C_{6}^{p n}(t)-k_{\mathrm{mat}} \cdot D C_{7}^{p n}(t)
$$

$$
\begin{aligned}
\frac{d L N D C_{7}^{p n}}{d t}= & k_{\mathrm{mat}} \cdot L N S \cdot E\left(\overline{a_{6}}\right) \cdot D C_{6}^{p n}(t) \\
& -k_{\mathrm{mat}} \cdot L N D C_{7}^{p n}(t)
\end{aligned}
$$

$$
\frac{d D C_{8}^{p n}}{d t}=k_{\mathrm{mat}} \cdot D C_{7}^{p n}(t)-k_{\mathrm{apop}} \cdot D C_{8}^{p n}(t)
$$

$$
\frac{d L N D C_{8}^{p n}}{d t}=k_{\mathrm{mat}} \cdot \operatorname{LNDC}_{7}^{p n}(t)-k_{\mathrm{apop}} \cdot \operatorname{LNDC}_{8}^{p n}(t)
$$

$$
\begin{gathered}
\frac{d D C_{1}^{n p}}{d t}=-k_{\mathrm{mat}} \cdot D C_{1}^{n p}(t)-k_{\mathrm{edu}}\left(\overline{a_{1}}\right) \cdot E S \cdot D C_{1}^{n p}(t) \\
\cdot f p 70+k_{\mathrm{edu}}\left(\overline{a_{1}}\right) \cdot E S \cdot D C_{1}^{n n}(t) \cdot f p 40
\end{gathered}
$$




$$
\begin{aligned}
\frac{d D C_{2}^{n p}}{d t}= & k_{\mathrm{mat}} \cdot D C_{1}^{n p}(t)-k_{\mathrm{mat}} \cdot D C_{2}^{p n}(t)-k_{\mathrm{edu}}\left(\overline{a_{2}}\right) \cdot E S \\
& \cdot D C_{2}^{n p}(t) \cdot f p 70+k_{\mathrm{edu}}\left(\overline{a_{2}}\right) \cdot E S \cdot D C_{2}^{n n}(t) \cdot f p 40
\end{aligned}
$$

$$
\begin{aligned}
\frac{d D C_{3}^{n p}}{d t}= & k_{\mathrm{mat}} \cdot D C_{2}^{n p}(t)-k_{\mathrm{mat}} \cdot D C_{3}^{p n}(t)-k_{\mathrm{edu}}\left(\overline{a_{3}}\right) \cdot E S \\
& \cdot D C_{3}^{n p}(t) \cdot f p 70+k_{\mathrm{edu}}\left(\overline{a_{3}}\right) \cdot E S \cdot D C_{3}^{n n}(t) \cdot f p 40
\end{aligned}
$$

$$
\begin{aligned}
\frac{d D C_{4}^{n p}}{d t}= & k_{\mathrm{mat}} \cdot D C_{3}^{n p}(t)-k_{\mathrm{mat}} \cdot D C_{4}^{p n}(t)-k_{\mathrm{edu}}\left(\overline{a_{4}}\right) \cdot E S \\
& \cdot D C_{4}^{n p}(t) \cdot f p 70+k_{\mathrm{edu}}\left(\overline{a_{4}}\right) \cdot E S \cdot D C_{4}^{n n}(t) \cdot f p 40
\end{aligned}
$$

$$
\begin{aligned}
\frac{d D C_{5}^{n p}}{d t}= & k_{\mathrm{mat}} \cdot D C_{4}^{n p}(t)-k_{\mathrm{mat}} \cdot D C_{5}^{p n}(t)-k_{\mathrm{edu}}\left(\overline{a_{5}}\right) \cdot E S \\
& \cdot D C_{5}^{n p}(t) \cdot f p 70+k_{\mathrm{edu}}\left(\overline{a_{5}}\right) \cdot E S \cdot D C_{5}^{n n}(t) \cdot f p 40
\end{aligned}
$$

$$
\begin{aligned}
\frac{d D C_{6}^{n p}}{d t}= & k_{\mathrm{mat}} \cdot D C_{5}^{n p}(t)-k_{\mathrm{mat}} \cdot\left[1-E\left(\overline{a_{6}}\right)\right] \cdot D C_{6}^{n p}(t) \\
& -k_{\mathrm{mat}} \cdot E\left(\overline{a_{6}}\right) \cdot D C_{6}^{n p}(t)-k_{\mathrm{edu}}\left(\overline{a_{6}}\right) \cdot E S \\
& \cdot D C_{6}^{n p}(t) \cdot f p 70+k_{\mathrm{edu}}\left(\overline{a_{6}}\right) \cdot E S \cdot D C_{6}^{n n}(t) \cdot f p 40
\end{aligned}
$$

$$
\frac{d D C_{7}^{n p}}{d t}=k_{\mathrm{mat}} \cdot\left[1-E\left(\overline{a_{6}}\right)\right] \cdot D C_{6}^{n p}(t)-k_{\mathrm{mat}} \cdot D C_{7}^{n p}(t)
$$

$$
\begin{aligned}
& \frac{d L N D C_{7}^{n p}}{d t}=k_{\mathrm{mat}} \cdot L N S \cdot E\left(\overline{a_{6}}\right) \cdot D C_{6}^{n p}(t) \\
& -k_{\mathrm{mat}} \cdot \operatorname{LNDC}_{7}^{n p}(t)
\end{aligned}
$$

$$
\frac{d D C_{8}^{n p}}{d t}=k_{\mathrm{mat}} \cdot D C_{7}^{n p}(t)-k_{\mathrm{apop}} \cdot D C_{8}^{n p}(t)
$$

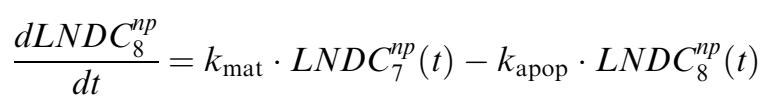

$$
\begin{aligned}
\frac{d D C_{1}^{p p}}{d t}= & -k_{\mathrm{mat}} \cdot D C_{1}^{p p}(t)+k_{\mathrm{edu}}\left(\overline{a_{1}}\right) \cdot E S \cdot D C_{1}^{n p}(t) \\
& \cdot f p 70+k_{\mathrm{edu}}\left(\overline{a_{1}}\right) \cdot E S \cdot D C_{1}^{p n}(t) \cdot f p 40
\end{aligned}
$$

$$
\begin{aligned}
\frac{d D C_{2}^{p p}}{d t}= & k_{\mathrm{mat}} \cdot D C_{1}^{p p}(t)-k_{\mathrm{mat}} \cdot D C_{2}^{p n}(t)+k_{\mathrm{edu}}\left(\overline{a_{2}}\right) \cdot E S \\
& \cdot D C_{2}^{n p}(t) \cdot f p 70+k_{\mathrm{edu}}\left(\overline{a_{2}}\right) \cdot E S \cdot D C_{2}^{p n}(t) \cdot f p 40
\end{aligned}
$$

$$
\begin{aligned}
\frac{d D C_{3}^{p p}}{d t}= & k_{\mathrm{mat}} \cdot D C_{2}^{p p}(t)-k_{\mathrm{mat}} \cdot D C_{3}^{p n}(t)+k_{\mathrm{edu}}\left(\overline{a_{3}}\right) \cdot E S \\
& \cdot D C_{3}^{n p}(t) \cdot f p 70+k_{\mathrm{edu}}\left(\overline{a_{3}}\right) \cdot E S \cdot D C_{3}^{p n}(t) \cdot f p 40
\end{aligned}
$$

$$
\begin{aligned}
\frac{d D C_{4}^{p p}}{d t}= & k_{\mathrm{mat}} \cdot D C_{3}^{p p}(t)-k_{\mathrm{mat}} \cdot D C_{4}^{p n}(t)+k_{\mathrm{edu}}\left(\overline{a_{4}}\right) \cdot E S \\
& \cdot D C_{4}^{n p}(t) \cdot f p 70+k_{\mathrm{edu}}\left(\overline{a_{4}}\right) \cdot E S \cdot D C_{4}^{p n}(t) \cdot f p 40
\end{aligned}
$$

$$
\begin{aligned}
\frac{d D C_{5}^{p p}}{d t}= & k_{\mathrm{mat}} \cdot D C_{4}^{p p}(t)-k_{\mathrm{mat}} \cdot D C_{5}^{p n}(t)+k_{\mathrm{edu}}\left(\overline{a_{5}}\right) \cdot E S \\
& \cdot D C_{5}^{n p}(t) \cdot f p 70+k_{\mathrm{edu}}\left(\overline{a_{5}}\right) \cdot E S \cdot D C_{5}^{p n}(t) \cdot f p 40
\end{aligned}
$$

$$
\begin{aligned}
\frac{d D C_{6}^{p p}}{d t}= & k_{\mathrm{mat}} \cdot D C_{5}^{p p}(t)-k_{\mathrm{mat}} \cdot\left[1-E\left(\overline{a_{6}}\right)\right] \cdot D C_{6}^{n p}(t) \\
& -k_{\mathrm{mat}} \cdot E\left(\overline{a_{6}}\right) \cdot D C_{6}^{n p}(t)+k_{\mathrm{edu}}\left(\overline{a_{6}}\right) \cdot E S \\
& \cdot D C_{6}^{n p}(t) \cdot f p 70+k_{\mathrm{edu}}\left(\overline{a_{6}}\right) \cdot E S \cdot D C_{6}^{p n}(t) \cdot f p 40
\end{aligned}
$$

$$
\frac{d D C_{7}^{p p}}{d t}=k_{\mathrm{mat}} \cdot\left[1-E\left(\overline{a_{6}}\right)\right] \cdot D C_{6}^{p p}(t)-k_{\mathrm{mat}} \cdot D C_{7}^{p p}(t)
$$

$$
\begin{aligned}
\frac{d L N D C_{7}^{p p}}{d t}= & k_{\mathrm{mat}} \cdot L N S \cdot E\left(\overline{a_{6}}\right) \cdot D C_{6}^{p p}(t) \\
& -k_{\mathrm{mat}} \cdot L N D C_{7}^{p p}(t)
\end{aligned}
$$

$$
\frac{d D C_{8}^{p p}}{d t}=k_{\mathrm{mat}} \cdot D C_{7}^{p p}(t)-k_{\mathrm{apop}} \cdot D C_{8}^{p p}(t)
$$

$$
\frac{d L N D C_{8}^{p p}}{d t}=k_{\mathrm{mat}} \cdot L N D C_{7}^{p p}(t)-k_{\mathrm{apop}} \cdot \operatorname{LNDC}_{8}^{p p}(t)
$$

where $f p 40$ and $f p 70$ are the "Total p40 Education" and "IL12p70 Education" relationships shown in Eqs. (6) and (7), respectively. For an acute antigen challenge protocol, the concentration of antigen is described by 


$$
\begin{aligned}
\frac{d A G}{d t}= & \operatorname{erf}\left[G_{\mathrm{Ag}} \cdot\left(t-t_{\mathrm{Agon}}\right)\right]-\operatorname{erf}\left[G_{\mathrm{Ag}} \cdot\left(t-t_{\mathrm{Agoff}}\right)\right] \\
& -k_{\mathrm{Agdeg}} \cdot A G(t),
\end{aligned}
$$

where the error functions approximate a step-function of height 1 starting at $t_{\text {Agon }}$ and ending at $t_{\text {Agoff }}$. The rate constant $k_{\text {Agdeg }}$ parameterizes the decay rate in antigen concentration. Values for the parameters are shown in Table 2. When challenged with an inflammatory mediator simultaneously with an antigen challenge, the concentration of IL-4, IFN- $\gamma$, and PGE2 are similarly described by

$$
\begin{aligned}
\frac{d I L 4}{d t}= & \operatorname{erf}\left[G_{\mathrm{Med}} \cdot\left(t-t_{\text {Medon }}\right)\right] \\
& -\operatorname{erf}\left[G_{\mathrm{Med}} \cdot\left(t-t_{\text {Medoff }}\right)\right]-k_{\text {Meddec }} \cdot I L 4(t)
\end{aligned}
$$

$$
\begin{aligned}
\frac{d I F N-\gamma}{d t}= & \operatorname{erf}\left[G_{\mathrm{Med}} \cdot\left(t-t_{\text {Medon }}\right)\right] \\
& -\operatorname{erf}\left[G_{\mathrm{Med}} \cdot\left(t-t_{\text {Medoff }}\right)\right]-k_{\mathrm{Meddec}} \cdot I F N-\gamma(t)
\end{aligned}
$$

$$
\begin{aligned}
\frac{d P G E 2}{d t}= & \operatorname{erf}\left[G_{\mathrm{Med}} \cdot\left(t-t_{\text {Medon }}\right)\right] \\
& -\operatorname{erf}\left[G_{\mathrm{Med}} \cdot\left(t-t_{\text {Medoff }}\right)\right]-k_{\text {Meddec }} \cdot P G E 2(t),
\end{aligned}
$$

where the rate constant $k_{\text {Meddec }}$ parameterizes the decay rate in inflammatory mediator concentration. Similarly, the parameters $t_{\text {Medon }}$ and $t_{\text {Medoff }}$ specify the start and end of a step-function. These values were selected to represent the dynamics of IL-4 expression observed by Batra et al. ${ }^{3}$

Following from the schematic diagram for tracking a cumulative characteristic of $D C_{i}$, antigen uptake and tracking for each of the $D C_{i}$ are given by the following equations:

$$
\begin{aligned}
\frac{d A G_{D C 1}}{d t}= & -k_{\mathrm{mat}} \cdot A G_{D C 1}(t)+k_{\text {uptake }}\left(\overline{a_{1}}\right) \cdot A G(t) \\
& \cdot\left(D C_{1}^{n n}(t)+D C_{1}^{p n}(t)+D C_{1}^{n p}(t)+D C_{1}^{p p}(t)\right)
\end{aligned}
$$

$$
\begin{aligned}
\frac{d A G_{D C 2}}{d t}= & k_{\text {mat }} \cdot A G_{D C 1}(t)-k_{\mathrm{mat}} \cdot A G_{D C 2}(t) \\
& +k_{\text {uptake }}\left(\overline{a_{2}}\right) \cdot A G(t) \cdot\left(D C_{2}^{n n}(t)\right. \\
& \left.+D C_{2}^{p n}(t)+D C_{2}^{n p}(t)+D C_{2}^{p p}(t)\right)
\end{aligned}
$$

$$
\begin{aligned}
\frac{d A G_{D C 3}}{d t}= & k_{\mathrm{mat}} \cdot A G_{D C 2}(t)-k_{\mathrm{mat}} \cdot A G_{D C 3}(t) \\
& +k_{\text {uptake }}\left(\overline{a_{3}}\right) \cdot A G(t) \cdot\left(D C_{3}^{n n}(t)\right. \\
& \left.+D C_{3}^{p n}(t)+D C_{3}^{n p}(t)+D C_{3}^{p p}(t)\right) \\
\frac{d A G_{D C 4}}{d t}= & k_{\mathrm{mat}} \cdot A G_{D C 3}(t)-k_{\mathrm{mat}} \cdot A G_{D C 4}(t) \\
& +k_{\text {uptake }}\left(\overline{a_{4}}\right) \cdot A G(t) \cdot\left(D C_{4}^{n n}(t)\right. \\
& \left.+D C_{4}^{p n}(t)+D C_{4}^{n p}(t)+D C_{4}^{p p}(t)\right)
\end{aligned}
$$

$$
\begin{aligned}
\frac{d A G_{D C 5}}{d t}= & k_{\mathrm{mat}} \cdot A G_{D C 4}(t)-k_{\mathrm{mat}} \cdot A G_{D C 5}(t) \\
& +k_{\text {uptake }}\left(\overline{a_{5}}\right) \cdot A G(t) \cdot\left(D C_{5}^{n n}(t)+D C_{5}^{p n}(t)\right. \\
& \left.+D C_{5}^{n p}(t)+D C_{5}^{p p}(t)\right)
\end{aligned}
$$

$$
\begin{aligned}
\frac{d A G_{D C 6}}{d t}= & k_{\mathrm{mat}} \cdot A G_{D C 5}(t)-k_{\mathrm{mat}} \cdot A G_{D C 6}(t) \\
& +k_{\text {uptake }}\left(\overline{a_{6}}\right) \cdot A G(t) \cdot\left(D C_{6}^{n n}(t)+D C_{6}^{p n}(t)\right. \\
& \left.+D C_{6}^{n p}(t)+D C_{6}^{p p}(t)\right)
\end{aligned}
$$

$$
\begin{aligned}
\frac{d A G_{D C 7}}{d t}= & k_{\text {mat }} \cdot\left[1-E\left(\overline{a_{6}}\right)\right] \cdot A G_{D C 6}(t)-k_{\text {mat }} \cdot A G_{D C 7}(t) \\
& +k_{\text {uptake }}\left(\overline{a_{7}}\right) \cdot A G(t) \cdot\left(D C_{7}^{n n}(t)+D C_{7}^{p n}(t)\right. \\
& \left.+D C_{7}^{n p}(t)+D C_{7}^{p p}(t)\right)
\end{aligned}
$$

$\frac{d A G_{L N D C 7}}{d t}=k_{\mathrm{mat}} \cdot E\left(\overline{a_{6}}\right) \cdot A G_{D C 6}(t)-k_{\mathrm{mat}} \cdot A G_{L N D C 7}(t)$

$$
\begin{aligned}
& \frac{d A G_{D C 8}}{d t}=k_{\mathrm{mat}} \cdot A G_{D C 7}(t)-k_{\mathrm{apop}} \cdot A G_{D C 8}(t) \quad(\mathrm{A} .55) \\
& \frac{d A G_{L N D C 8}}{d t}=k_{\mathrm{mat}} \cdot A G_{L N D C 7}(t)-k_{\mathrm{apop}} \cdot A G_{L N D C 8}(t)
\end{aligned}
$$

The following equations describe the rate of change of IL12p70, IL12p40, and IL12(p40) 2 in the lymph node:

$$
\begin{aligned}
\frac{d I L 12 p 40}{d t}= & (1-F D) \times B M R p 40 p 70 \times M W p 70 \\
& \times C P p 70 \times\left(L N D C_{7}^{n p}(t)+L N D C_{7}^{p p}(t)\right) \\
& -k_{\text {Meddec }} \times I L 12 p 40(t)
\end{aligned}
$$




$$
\begin{aligned}
\frac{d I L 12(p 40)_{2}}{d t}= & 1 / 2 \times F D \times B M R p 40 p 70 \times M W p 70 \\
& \times C P p 70 \times\left(L N D C_{7}^{n p}(t)+L N D C_{7}^{p p}(t)\right) \\
& -k_{\text {Meddec }} \times I L 12(p 40)_{2}(t)
\end{aligned}
$$

$$
\begin{aligned}
\frac{d I L 12 p 70}{d t}= & M W p 70 \times C P p 70 \times\left(\operatorname{LNDC}_{7}^{p n}(t)\right. \\
& \left.+L N D C_{7}^{p p}(t)\right)-k_{\mathrm{Meddec}} \times I L 12 p 70(t)
\end{aligned}
$$

The following equations provide an estimate of net IL-12 bioactivity based on the competitive interaction of IL12p40, IL12(p40) 2 , and IL12p70 with the IL-12 receptor:

$$
\begin{aligned}
& F O_{p 70}= \\
& I L 12 p 70(t)+\frac{K_{D_{p} 70}}{K_{p 40}} \cdot I L 12 p 40(t)+\frac{K_{D p 70}}{K_{(p 40) 2}} \cdot I L 12(p 40)_{2}(t)+K_{D p 70}
\end{aligned}
$$

$$
\text { IL12 Bioactivity }=\frac{S D \times F O_{p 70}}{S D \times F O_{p 70}+K_{M}},
$$

where $F O_{p 70}$ is the fractional occupancy of the IL-12 receptor.

\section{ACKNOWLEDGMENTS}

The author declares no competing financial interest. The author would like to thank Profs. C. Cuff (Microbiology, Immunology, \& Cell Biology, WVU) and J. Shaeiwitz (Chemical Engineering, WVU), and the anonymous referees for their critical reading of this manuscript. This work was supported by the West Virginia University Research Corporation.

\section{REFERENCES}

\footnotetext{
${ }^{1}$ Abbas, A. K., and C. A. Janeway Jr. Immunology: Improving on nature in the twenty-first century. Cell 100:129-138, 2000.

${ }^{2}$ Banchereau, J., F. Briere, C. Caux, J. Davoust, S. Lebecque, Y. J. Liu, B. Pulendran, and K. Palucka. Immunobiology of dendritic cells. Annu. Rev. Immunol. 18:767-811, 2000.

${ }^{3}$ Batra, V., A. I. Musani, A. T. Hastie, S. Khurana, K. A. Carpenter, J. G. Zangrilli, and S. P. Peters. Bronchoalveolar lavage fluid concentrations of transforming growth factor (TGF)- $\beta 1$, TGF- $\beta 2$, Interleukin (IL)- 4 and IL-13 after segmental allergen challenge and their effects on $\alpha$ smooth muscle actin and collagen III synthesis by primary
}

human lung fibroblasts. Clin. Exp. Allergy 34:437-444, 2004.

${ }^{4}$ Catron, D. M., A. A. Itano, K. A. Pape, D. L. Mueller, and M. K. Jenkins. Visualizing the first $50 \mathrm{hr}$ of the primary immune response to a soluble antigen. Immunity 21:341-347, 2004

${ }^{5}$ Caux, C., C. Massacrier, B. Vanbervliet, B. Dubois, I. Durand, M. Cella, A. Lanzavecchia, and J. Banchereau. $\mathrm{CD} 34+$ hematopoietic progenitors from human cord blood differentiate along two independent dendritic cell pathways in response to granulocyte-macrophage colonystimulating factor plus tumor necrosis factor alpha: II. Functional analysis. Blood 90:1458-1470, 1997.

${ }^{6}$ Desai, B. B., P. M. Quinn, A. G. Wolitzky, P. K. Mongini, R. Chizzonite, and M. K. Gately. IL-12 receptor. II. Distribution and regulation of receptor expression. J. Immunol. 148:3125-3132, 1992.

${ }^{7}$ Dzionek, A., A. Fuchs, P. Schmidt, S. Cremer, M. Zysk, S. Miltenyi, D. W. Buck, and J. Schmitz. BDCA-2, BDCA-3, and BDCA-4: three markers for distinct. subsets of dendritic cells in human peripheral blood. J. Immunol. 165:6037-6046, 2000.

${ }^{8}$ Ebner, S., G. Ratzinger, B. Krosbacher, M. Schmuth, A. Weiss, D. Reider, R. A. Kroczek, M. Herold, C. Heufler, P. Fritsch, and N. Romani. Production of IL-12 by human monocyte-derived dendritic cells is optimal when the stimulus Is given at the onset of maturation, and Is further enhanced by IL-4. J. Immunol. 166:633-641, 2001.

${ }^{9}$ Elgeti, K. A new equation for correlating a pipe flow reactor with a cascade of mixed reactors. Chem. Eng. Sci. 51:5077-5080, 1996.

${ }^{10}$ FDA. Innovation or Stagnation: Challenge and Opportunity on the Critical Path to New Medical Products. U.S. Department of Health and Human Services, Food and Drug Administration, 2004. URL http://www.fda.gov/oc/ initiatives/criticalpath/whitepaper.pdf.

${ }^{11}$ Fecek, R. J., R. Busch, H. Lin, K. Pal, C. A. Cunningham, and C. F. Cuff. Production of Alexa Fluor 488-labeled reovirus and characterization of target cell binding, competence, and immunogenicity of labeled virions. J. Immunol. Methods 314:30-37, 2006.

${ }^{12}$ Fujii, S., K. Liu, C. Smith, A. J. Bonito, and R. M. Steinman. The linkage of innate to adaptive immunity via maturing dendritic cells in vivo requires CD40 ligation in addition to antigen presentation and CD80/86 costimulation. J. Exp. Med. 199:1607-1618, 2004.

${ }^{13}$ Gately, M. K., L. M. Renzetti, J. Magram, A. S. Stern, L. Adorini, U. Gubler, and D. H. Presky. The Interleukin-12/ Interleukin-12-receptor system: role in normal and pathologic immune responses. Annu. Rev. Immunol. 16:495-521, 1998.

${ }^{14}$ Germain, R. N. The art of the probable: system control in the adaptive immune system. Science 293:240-245, 2001.

${ }^{15}$ Heinzel, F. P., A. M. Hujer, F. N. Ahmed, and R. M. Rerko. In vivo production and function of IL-12 p40 homodimers. J. Immunol. 158:4381-4388, 1997.

${ }^{16}$ Herpen, C. M. V., R. Huijbens, M. Looman, J. de Vries, H. Marres, D. Van, R. Hermsen, G. J. Adema, and P. D. Mulder. Pharmacokinetics and immunological aspects of a phase Ib study with intratumoral administration of recombinant human Interleukin-12 in patients with head and neck squamous cell carcinoma: a decrease of $\mathrm{T}$-bet in peripheral blood mononuclear cells. Clin. Cancer Res. 9:2950-2956, 2003.

${ }^{17}$ Hochrein, H., M. O'Keeffe, T. Luft, S. Vandenabeele, R. J. Grumont, E. Maraskovsky, and K. Shortman. Interleukin 
(IL)-4 is a major regulatory cytokine governing bioactive IL-12 production by mouse and human dendritic cells. $J$. Exp. Med. 192:823-833, 2000.

${ }^{18}$ Huh, J. C., D. H. Strickland, F. L. Jahnsen, D. J. Turner, J. A. Thomas, S. Napoli, I. Tobagus, P. A. Stumbles, P. D. Sly, and P. G. Holt. Bidirectional interactions between antigen-bearing respiratory tract dendritic cells (DCs) and $\mathrm{T}$ cells precede the late phase reaction in experimental asthma: DC activation occurs in the airway mucosa but not in the lung parenchyma. J. Exp. Med. 198:19-30, 2003.

${ }^{19}$ Ingulli, E., A. Mondino, A. Khoruts, and M. K. Jenkins. In vivo detection of dendritic cell antigen presentation to CD4(+) T cells. J. Exp. Med. 185:2133-2141, 1997.

${ }^{20}$ Irish, J. M., R. Hovland, P. O. Krutzik, O. D. Perez, O. Bruserud, B. T. Gjertsen, and G. P. Nolan. Single cell profiling of potentiated phospho-protein networks in cancer cells. Cell 118:217-228, 2004.

${ }^{21}$ Jacobson, N. G., S. J. Szabo, R. M. Weber-Nordt, Z. Zhong, R. D. Schreiber, J. E. Darnell, and K. M. Murphy. Interleukin 12 signaling in T helper type 1 (Th1) cells involves tyrosine phosphorylation of signal transducer and activator of transcription (Stat)3 and Stat4. J. Exp. Med. 181:1755-1762, 1995.

${ }^{22}$ Jahnsen, F. L., E. D. Moloney, T. Hogan, J. W. Upham, C. M. Burke, and P. G. Holt. Rapid dendritic cell recruitment to the bronchial mucosa of patients with atopic asthma in response to local allergen challenge. Thorax 56:823-826, 2001.

${ }^{23}$ Janeway, C. A., P. Travers, M. Walport, and M. Shlomchik. Immunobiology: The Immune System in Health and Disease. New York: Garland Publishing, 2001, 732 pp.

${ }^{24}$ Jenkins, M. K., A. Khoruts, E. Ingulli, D. L. Mueller, S. J. McSorley, R. L. Reinhardt, A. Itano, and K. A. Pape. In vivo activation of antigen-specific CD4 T cells. Annu. Rev. Immunol. 19:23-45, 2001.

${ }^{25}$ Kalinski, P., C. M. Hilkens, E. A. Wierenga, and M. L. Kapsenberg. T-cell priming by type- 1 and type- 2 polarized dendritic cells: the concept of a third signal. Immunol. Today 20:561-567, 1999.

${ }^{26}$ Kalinski, P., J. H. Schuitemaker, C. M. Hilkens, and M. L. Kapsenberg. Prostaglandin E2 induces the final maturation of IL-12-deficient CD1a + CD83 + dendritic cells: the levels of IL-12 are determined during the final dendritic cell maturation and are resistant to further modulation. $J$. Immunol. 161:2804-2809, 1998.

${ }^{27}$ Kalinski, P., P. L. Vieira, J. H. Schuitemaker, E. C. de Jong, and M. L. Kapsenberg. Prostaglandin E(2) is a selective inducer of Interleukin-12 p40 (IL-12p40) production and an inhibitor of bioactive IL-12p70 heterodimer. Blood 97:3466-3469, 2001.

${ }^{28}$ Khoo, M. C. K. Physiological Control Systems: Analysis, Simulation, and Estimation. Piscataway, NJ: IEEE Press Series on Biomedical Engineering, 2000.

${ }^{29}$ Klinke, D. J. An age-structured model of dendritic cell trafficking in the lung. Am. J. Physiol. Lung Cell Mol. Physiol. 291:L1038-L1049, 2006.

${ }^{30}$ Klinke, D. J. The ratio of $\mathrm{P} 40$ monomer to dimer is an important determinant of IL-12 bioactivity. J. Theor. Biol. 240:323-335, 2006

${ }^{31}$ Langenkamp, A., M. Messi, A. Lanzavecchia, and F. Sallusto. Kinetics of dendritic cell activation: impact on priming of Th1, Th2 and nonpolarized $\mathrm{T}$ cells. Nat. Immunol. 1:311-316, 2000.

${ }^{32}$ Ling, P., M. K. Gately, U. Gubler, A. S. Stern, P. Lin, K. Hollfelder, C. Su, Y. C. Pan, and J. Hakimi. Human IL-12 p40 homodimer binds to the IL-12 receptor but does not mediate biologic activity. J. Immunol. 154:116-127, 1995.

${ }^{33}$ Lorenzon, P., E. Vecile, E. Nardon, E. Ferrero, J. M. Harlan, F. Tedesco, and A. Dobrina. Endothelial cell Eand P-selectin and vascular cell adhesion molecule-1 function as signaling receptors. J. Cell Biol. 142:1381-1391, 1998.

${ }^{34}$ Luft, T., M. Jefford, P. Luetjens, T. Toy, H. Hochrein, K.A. Masterman, C. Maliszewski, K. Shortman, J. Cebon, and E. Maraskovsky. Functionally distinct dendritic cell (DC) populations induced by physiologic stimuli: prostaglandin E2 regulates the migratory capacity of specific DC subsets. Blood 100:1362-1372, 2002.

${ }^{35}$ Matzinger, P. The danger model: a renewed sense of self. Science 296:301-305, 2002.

${ }^{36}$ Medzhitov, R., and C. A. Janeway Jr. Innate immunity: the virtues of a nonclonal system of recognition. Cell 31:295298, 1997.

${ }^{37}$ Murphy, F. J., M. P. Hayes, and P. R. Burd. Disparate intracellular processing of human IL-12 preprotein subunits: atypical processing of the P35 signal peptide. $J$. Immunol. 164:839-847, 2000.

${ }^{38}$ Naito, T., Y. Ozawa, M. Tomoyasu, M. Inagaki, M. Fukue, M. Sakai, T. Yamamoto, S. Ishikawa, and M. Onizuka. New method for evaluation of lung lymph flow rate with intact lymphatics in anaesthetized sheep. Acta Physiol. 188:139-149, 2006.

${ }^{39}$ Ohno, R., Y. Yamaguchi, T. Toge, T. Kinouchi, T. Kotake, M. Shibata, Y. Kiyohara, S. Ikeda, I. Fukui, A. Gohchi, Y. Sugiyama, S. Saji, S. Hazama, M. Oka, K. Ohnishi, Y. Ohhashi, S. Tsukagoshi, and T. Taguchi. A dose-escalation and pharmacokinetic study of subcutaneously administered recombinant human Interleukin 12 and its biological effects in Japanese patients with advanced malignancies. Clin. Cancer Res. 6:2661-2669, 2000.

${ }^{40}$ O'Neill, D. W., S. Adams, and N. Bhardwaj. Manipulating dendritic cell biology for the active immunotherapy of cancer. Blood 104:2235-2246, 2004.

${ }^{41}$ Ottino, J. M. New tools, new outlooks, new opportunities. AIChE J. 51:1840-1845, 2005.

${ }^{42}$ Park, G. Y., and J. W. Christman. Involvement of cyclooxygenase-2 and prostaglandins in the molecular pathogenesis of inflammatory lung diseases. Am. J. Physiol. Lung Cell. Mol. Physiol. 290:797-805, 2006.

${ }^{43}$ Presky, D. H., L. J. Minetti, S. Gillessen, V. L. Wilkinson, C. Y. Wu, U. Gubler, R. Chizzonite, and M. Gately. Analysis of the multiple interactions between IL-12 and the high affinity IL-12 receptor complex. J. Immunol. 160:2174-2179, 1998.

${ }^{44}$ Rich, I. N. Monocytes and Macrophages. In: Human Cell Culture, Vol. 4, Primary Hematopoietic Cells, edited by M. R. Koller and B. O. Palsson. Kluwer Academic Publishers, 1999, pp. 125-145.

${ }^{45}$ Seder, R. A., and W. E. Paul. Acquisition of lymphokineproducing phenotype by CD $4+\mathrm{T}$ cells. Annu. Rev. Immunol. 12:635-673, 1994.

${ }^{46}$ Smith, K. A. The quantal theory of how the immune system discriminates between "self and non-self". Med. Immunol. 3:3, 2004.

${ }^{47}$ Snijders, A., P. Kalinski, C. M. Hilkens, and M. L. Kapsenberg. High-level IL-12 production by human dendritic cells requires two signals. Int. Immunol. 10:1593-1598, 1998.

${ }^{48}$ Sozzani, S. Dendritic cell trafficking: more than just chemokines. Cytokine Growth Factor Rev. 16:581-592, 2005. 
${ }^{49}$ Suzuki, H., M. Takei, M. Yanagida, T. Nakahata, T. Kawakami, and H. Fukamachi. Early and late events in FceRl signal transduction in human cultured mast cells. $J$. Immunol. 159:5881-5888, 1997.

${ }^{50}$ Thompson, C. B. New insights into V(D)J recombination and its role in the evolution of the immune system. Immunity 3:531-539, 1995.

${ }^{51}$ Trinchieri, G. Interleukin-12 and the regulation of innate resistance and adaptive immunity. Nat. Rev. Immunol. 3:133-146, 2003.

${ }^{52}$ Tsurui, H., H. Nishimura, S. Hattori, S. Hirose, K. Okumura, and T. Shirai. Seven-color fluorescence imaging of tissue samples based on Fourier spectroscopy and singular value decomposition. J. Histochem. Cytochem. 48:653-662, 2000.

${ }^{53}$ Upham, J. W., J. Lundahl, H. Liang, J. A. Denburg, P. M. O'Byrne, and D. P. Snider. Simplified quantitation of myeloid dendritic cells in peripheral blood using flow cytometry. Cytometry 40:50-59, 2000.
${ }^{54}$ Vermaelen, K. Y., I. Carro-Muino, B. N. Lambrecht, and R. A. Pauwels. Specific migratory dendritic cells rapidly transport antigen from the airways to the thoracic lymph nodes. J. Exp. Med. 193:51-60, 2001.

${ }^{55}$ Vermaelen, K. Y., and R. A. Pauwels. Pulmonary dendritic cells. Am. J. Respir. Crit. Care Med. 172:530-551, 2005.

${ }^{56}$ Vlachos, D. G. A review of multiscale analysis: examples from systems biology, materials engineering, and other fluid-surface interacting systems. Adv. Chem. Eng. 30:1-61, 2005.

${ }^{57}$ Whitelaw, D. M. The intravascular lifespan of monocytes. Blood 28:455-464, 1966.

${ }^{58}$ Woodhead, V. E., M. H. Binks, B. M. Chain, and D. R. Katz. From sentinel to messenger: an extended phenotypic analysis of the monocyte to dendritic cell transition. Immunology 94:552-559, 1998.

${ }^{59}$ Yates, A., C. C. Chan, R. E. Callard, A. J. George, and J. Stark. An approach to modelling in immunology. Brief Bioinform. 2:245-257, 2001. 